\title{
UNMANNED AERIAL VEHICLES FOR POST DISASTER SURVEYS
}

\author{
A Thesis \\ presented to \\ the Faculty of California Polytechnic State University, \\ San Luis Obispo \\ In Partial Fulfillment \\ of the Requirements for the Degree \\ Master of Science in Architecture
}

by

Thor Liland Larsen

December 2010 
(C) 2010

Thor Liland Larsen

ALL RIGHTS RESERVED 


\section{COMMITTEE MEMBERSHIP}

TITLE: $\quad$ Unmanned Aerial Vehicles for Post Disaster Surveys

AUTHOR: $\quad$ Thor Liland Larsen

DATE SUBMITTED: December 2010

COMMITTEE CHAIR: Professor Jens Pohl, Graduate Coordinator, Architecture Department

COMMITTEE MEMBER: Professor Arthur Chapman, Architecture Department

COMMITTEE MEMBER: Dr. David Niebuhr, Lecturer, Physics Department 


\section{ABSTRACT \\ Unmanned Aerial Vehicles for Post Disaster Surveys}

Thor Liland Larsen

In the current built environment, structures require regular observation and maintenance. Many of these structures can be quite challenging to evaluate. The required scaffolding, lifts, or similar access facilities can become quite costly to rent and construct, and can be a long term disturbance to those who use and manage the particular structure. Furthermore, there are situations where examination for the purpose of detailed analysis can be quite hazardous, if not entirely unsafe for humans. In a post-disaster environment traditional methods may not be safe or adequate for gaining access to parts of a structure that require observation or analysis. The use of a remotely controlled unmanned vehicle is a reliable, safe and cost effective substitute for assessing structures before and after seismic, terrorist, or other destructive events.

Keywords: UAV, survey, unmanned, remote control, post disaster, structure 


\section{ACKNOWLEDGEMENTS}

I would like to thank my wife Harmony, and my parents Anne-Marie and Robert, for the support they offered while I was writing this thesis. Additionally, I would like to thank Dr. Jens Pohl for his belief that I could complete this project, and his tireless proofreading. Lastly I would like to thank Dr. David Niebuhr and Professor Arthur Chapman for accepting the role as committee members. Without the aid of all these individuals this project would not have been completed. 


\section{TABLE OF CONTENTS}

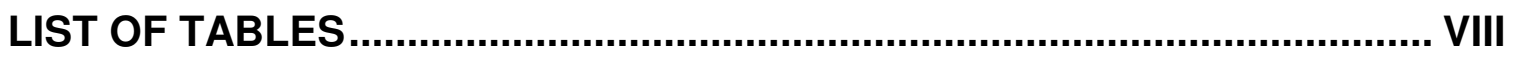

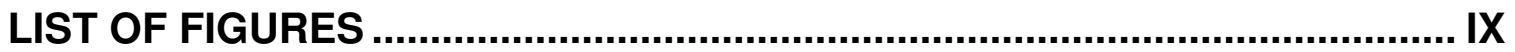

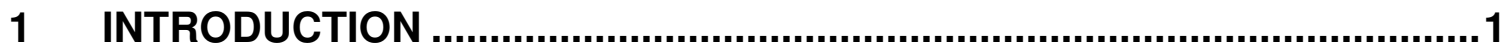

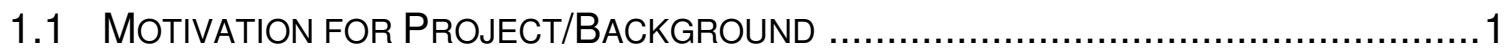

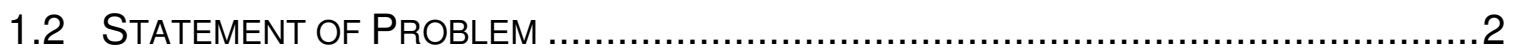

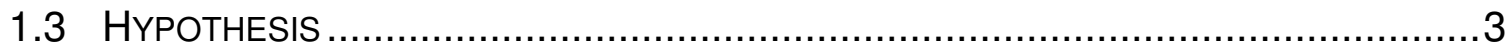

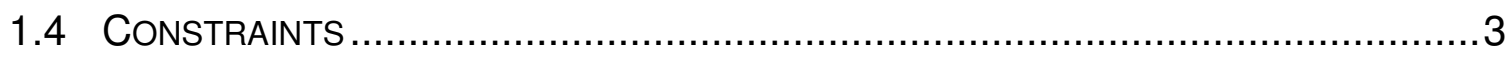

2 BACKGROUND ON VEHICLE TYPES ....................................................4

2.1 Helicopters, Non-Coaxial Multi-Rotors, Airplanes, OR Lighter-Than-

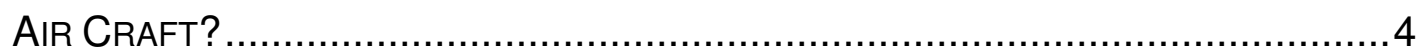

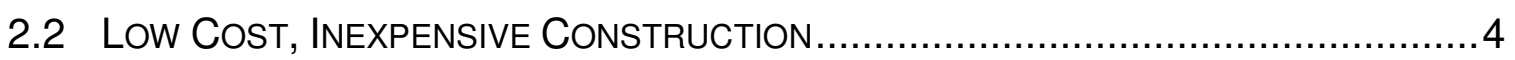

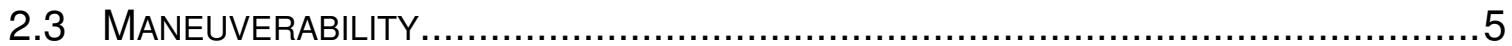

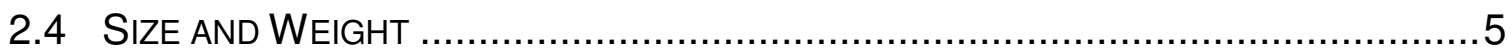

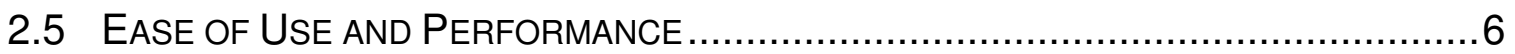

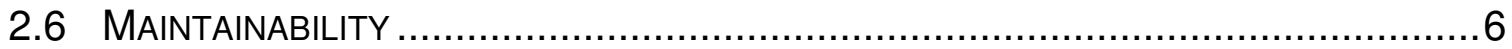

2.7 AdVANTAGES AND DISADVANTAGES OF EACH VEHICLE TYPE ............................

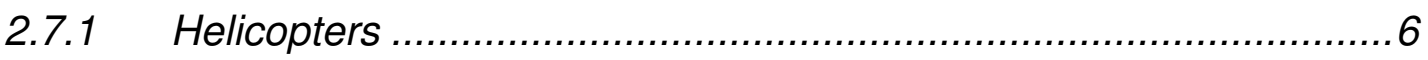

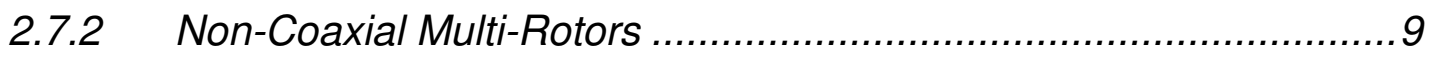

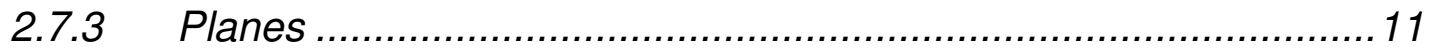

2.7.4 Lighter-Than-Air Craft_...................................................... 13

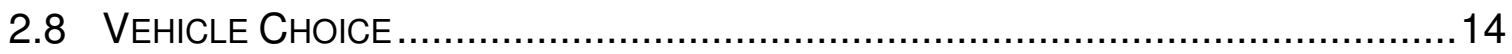

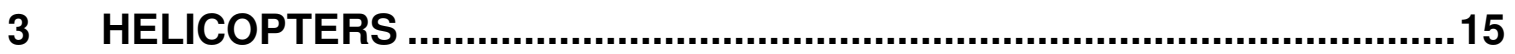

3.1 Operational Characteristics of a Helicopter …................................15

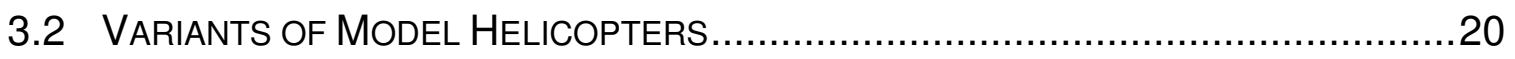

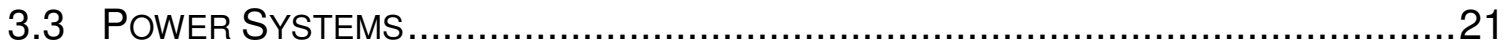

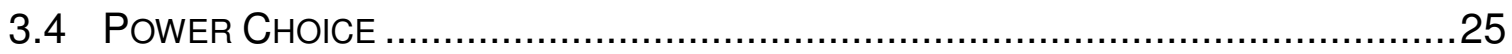


4 SELECTING AND BUILDING THE AERIAL PLATFORM .......................26

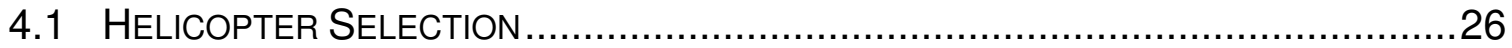

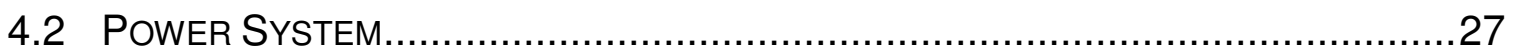

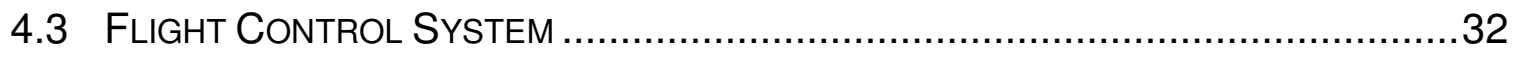

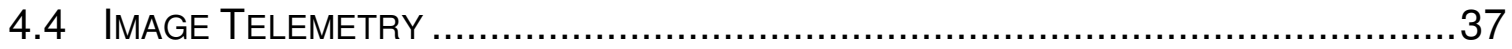

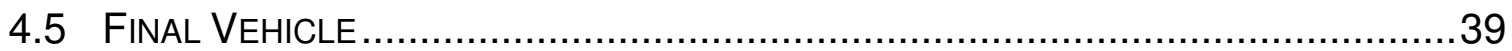

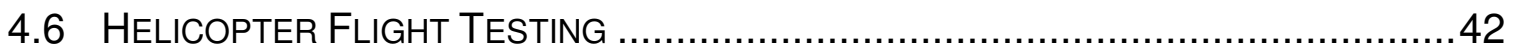

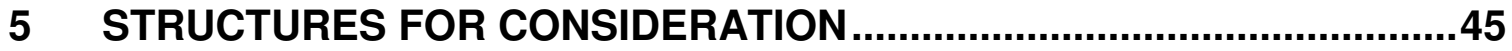

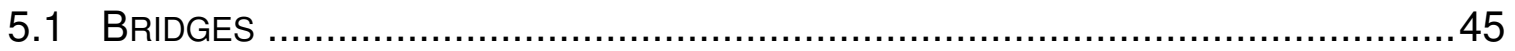

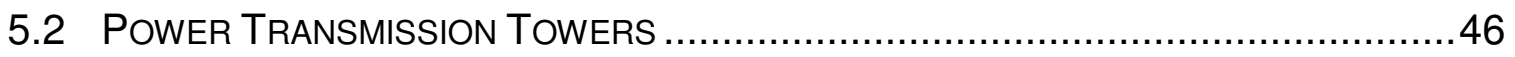

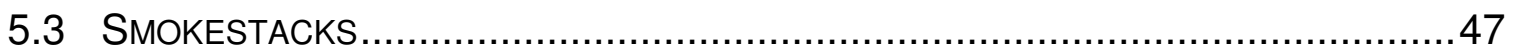

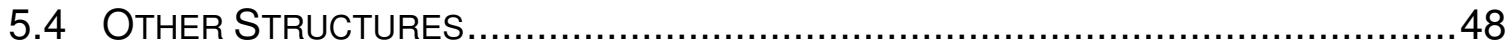

6 CRITERIA FOR TEST SURVEY STRUCTURE ......................................50

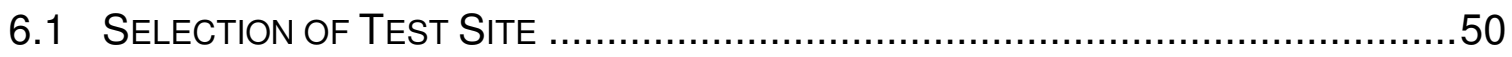

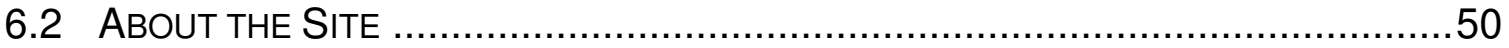

7 PRACTICAL HELICOPTER RESULTS ….............................................53

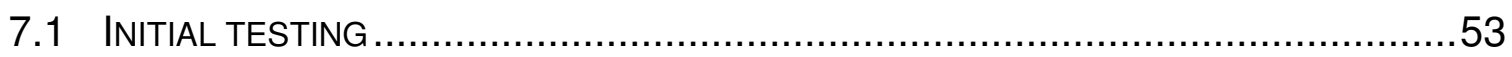

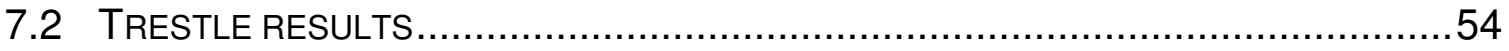

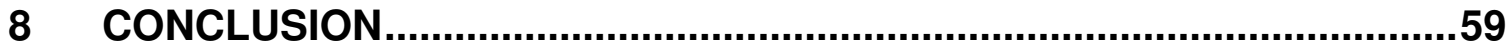

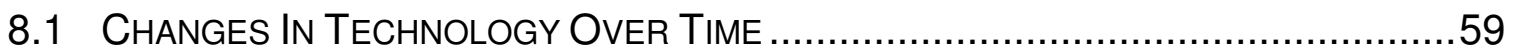

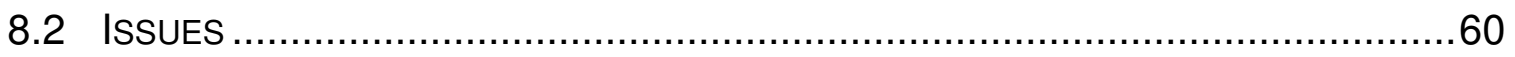

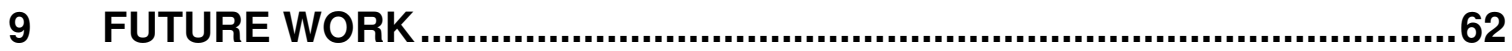

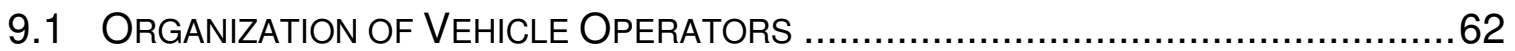

9.2 Avallable User Base in Model Aircraft Flying ......................................62

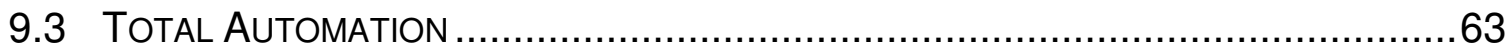

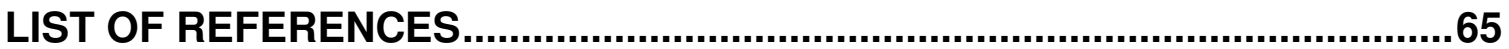




\section{LIST OF TABLES}

TABLE 4-1 COST OF VEHICLE COMPONENTS AS CURRENTLY BUILT. .........................40

TABLE 4-2 ESTIMATEd COST USING ALTERNATIVE OR LESS EXPENSIVE HARDWARE. ...41

TABle 9-1 Number of R/C Flying Clubs Distributed ACROSS THE U.S..............63 


\section{LIST OF FIGURES}

FiguRE 1-1 LIGHT STREAMING THROUGH HOLES IN THE LA, SUPERDOME ROOF. (C)

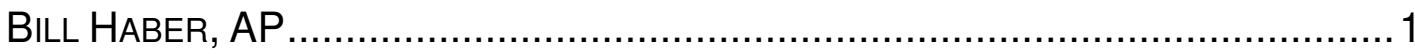

FIGURE 2-1, TYPICAL MODEL HELICOPTER IN FLIGHT. () HTTP://WWW.NITROPLANES.COM/RAG26CCRAREC.HTML ............................... 7

FIGURE 2-2 THIS IS AN EXAMPLE OF THE SWASH PLATE AND ROTOR HEAD CONTROL LINKAGES.

FIGURE 2-3 QUADCOPTER IN FLIGHT, ONE OF MANY VARIANTS OF NON-COAXIAL MULTI-ROTOR AIRCRAFT. (C) 2009, STEVENS INSTITUTE OF TECHNOLOGY 10

FIGURE 2-4 MULTIPLEX EASYSTAR, AN INEXPENSIVE, EASILY MODIFIABLE MODEL PLANE

FIGURE 2-5 A PAYLOAD TOTING BLIMP WITH BELLY CAMERA. @ RYAN WINSLOW,

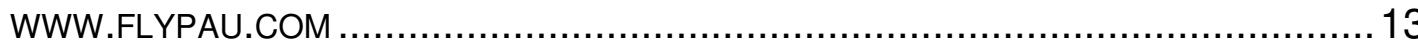

FIGURE 3-1, FUNDAMENTAL PARTS OF A HELICOPTER ....................................... 15

FIGURE 3-2 THIS GRAPHIC ILLUSTRATES THE TOROIDAL NATURE OF THE VORTEX RING AROUND THE OUTER EDGE OF THE MAIN ROTOR

Figure 4-1 SWIFT 16 By CENTURY HeLICOPTER AS IT APPEARS WHEN BUILT UNMODIFIED. (C) CentuRY HeLICOPTERS 26

FigURE 4-2 CENTURY 600, $715 \mathrm{KV}$ OUTRUNNER WITH ATTACHED COOLING ROTOR. ...28 FiguRE 4-3 MAXAMPS 10,000 MAH, 14.8 VOLT LIPO BATTERY. WEIGHT 820 GRAMS.

FIGURE 4-4 WATTAGE, AMPERAGE, CUMULATIVE MAH, AND BATTERY VOLTAGE DURING A 10 MINUTE FLIGHT WITH A 14.8 VOLT (FOUR CELL), 10,000 MAH BATTERY.

FiguRE 4-5 MAXAMPS 6,500 MAH 22.2 VOLt LIPO BATTERY. WEIGHT 951 GRAMS. .. 30

FIGURE 4-6 WATTAGE, AMPERAGE, CUMULATIVE MAH, AND BATTERY VOLTAGE DURING A 10 MINUTE FLIGHT WITH A 22.2 VOLT (SIX CELL), 6500 MAH BATTERY. . 31

Figure 4-7 Castle Creation "Phoenix HV45 ESC" electronic SPeEd CONTROLLER. 32

FiguRE 4-8 MULTIPLEX ROYAL EVo 12, 12 CHANNEL RADIO TRANSMITTER. 34 
FiguRE 4-9 HITEC SUPREME IIS 8 CHANNEL REMOTE CONTROL AIRCRAFT RECEIVER . 35

Figure 4-10 HeliCommand 3A Flight Augmentation Computer and INTERFACE.

FIGURE 4-11 BLACK WIDOW $2.4 \mathrm{GHZ}$ AUDIO/VIDEO TRANSMITTER AND RECEIVER. .....39

Figure 4-12Century HeLICOPTER, SWIFT 16 PREPPED FOR AERIAL PHOTOGRAPHY. INCLUDED ALSO IS THE BASE STATION, MULTIPLEX TRANSMITTER, AND THE AUDIO/VIDEO TELEMETRY BASE STATION

Figure 5-1 Kelly Creek Trestle, Montana, 260 meters long, 70 meters HIGH. AN EXAMPLE OF A BRIDGE STRUCTURE THAT COULD BENEFIT FROM QUICK, INEXPENSIVE, SURVEY TECHNIQUES.

FIGURE 5-2 THREE PHASE ALTERNATING CURRENT TRANSMISSION TOWERS.

FIGURE 5-3 MORRO BAY POWER PLANT WITH THREE 137 METER SMOKESTACKS. 48

Figure 6-1 The STENNER CREEK TRESTLE WITH AN INDICATION OF THE AREA THAT WAS CHOSEN FOR SURVEY. AERIAL PICTURE @ 2010 GoOgLe EARTH.

FIGURE 6-2 THE CONNECTION POINT OF THE TRESTLE AND THE GIRDER IS THE FOCAL POINT OF STUDY WITH THE REMOTE VEHICLE, AS VIEWED TO THE SOUTH SOUTHWEST, FROM ROAD LEVEL. 52

FIGURE 7-1 A PICTURE TAKEN FROM THE PHOTO PLATFORM PRIOR TO EDITING. 55

Figure 7-2 With Adobe PHOtOSHOP IT IS POSSIBLE TO ENHANCE THE PICTURE SO MORE DETAIL IS AVAILABLE. 55

FIGURE 7-3 ANOTHER IMAGE WITH MORE EXPOSED DETAIL. 56

FIGURE 7-4 THE VIEW OF A CROPPED AREA OF FIGURE 2-3 WITH SOME SLIGHT

PHOTOSHOP CONTRAST AND SHARPENING ADJUSTMENTS. 56

FIGURE 7-5 ANOTHER EXAMPLE IMAGE WITH BETTER EXPOSURE. 57

FIGURE 7-6 A CLOSE UP CROP AND ROTATED VERSION OF FIGURE 7-5, NOTE THE ORANGE FIBER OPTIC CABLE CONDUIT ON THE UPPER LEFT CENTER. 


\section{Introduction}

\subsection{Motivation for Project/Background}

In 2005 Hurricane Katrina struck New Orleans. The Superdome suffered a partial roof failure while it was being used as a gathering and shelter point. In Figure 1-1, beams of light where the sun was shining through broken skylights are clearly visible.

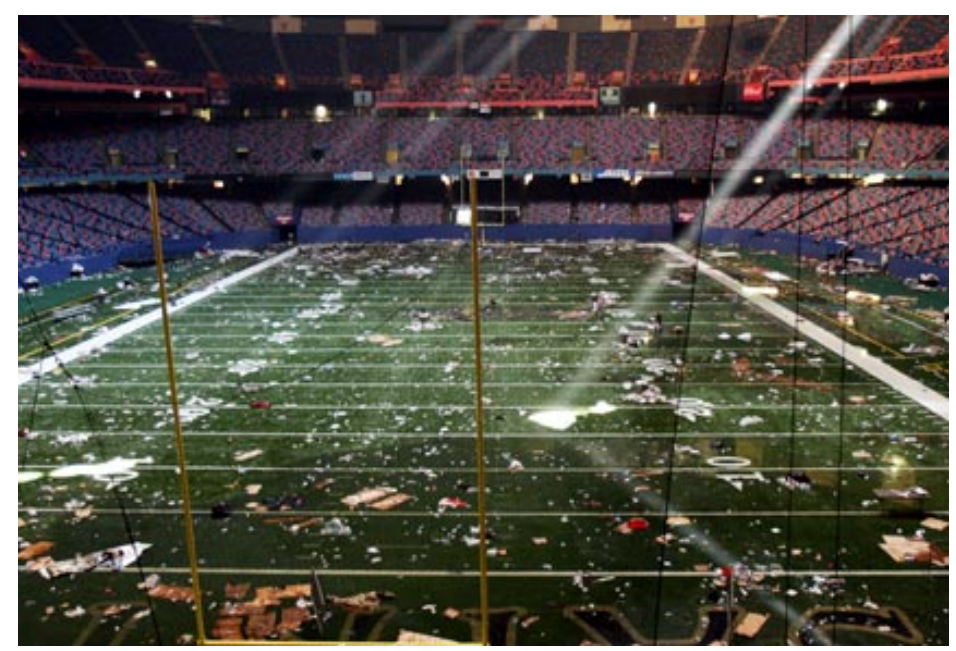

Figure 1-1 Light streaming through holes in the LA, Superdome roof. @ Bill Haber, AP

Until that moment, although involved in Remote Control $(\mathrm{R} / \mathrm{C})$ for some time, the author had not realized a potential for use of $\mathrm{R} / \mathrm{C}$ equipment for rapid and inexpensive damage assessment. There had been posted, to the Internet, a number of movies displaying various videos taken from airborne $\mathrm{R} / \mathrm{C}$ equipment, but they seemed to underutilize the capabilities available to them. Generally the video was either taken from too high an altitude to be useful, or the pilots just flew around, seemingly without a plan, but did not loiter or find anything that appeared significant or useful. 
It became apparent that in a world of radically changing weather, political turmoil, and terrorism, there exists a need for methods of rapid structural survey that will insulate and isolate investigators from the inherent dangers associated with failing structures. After exploration of methods currently employed for structural surveys, it became clear that there is room for another tool that can be deployed quickly and safely while yielding high quality imagery and aiding in a structural assessment.

\subsection{Statement of Problem}

The failure of structures due to natural or manmade disasters cannot realistically be eliminated despite the best efforts of designers and engineers. When an event occurs that may put structures at risk, and consequently put lives at risk, it is important to have technology available that can facilitate surveys of the structures as quickly and reliably as possible. Due to the various sizes, shapes, and physical locations of structures, it is not always as simple as taking ground based photos by bringing in cherry pickers, or finding full size planes or helicopters for use as observation platforms. Furthermore, in the case of a wide area event (i.e., earthquake, flood, heavy snowfalls, or other events) those resources may be employed for rescue, grounded, or otherwise unavailable.

It seems possible to create a remotely controlled vehicle from mostly generic, commercial off-the-shelf (COTS) components, to be employed as a tool to gather visual data for analysis and determination of safety and structural worthiness. Additionally, the low cost of assembly coupled with simplicity of operation should 
place the tool well within reach of the budget and usability constraints of any municipality.

\subsection{Hypothesis}

It is possible to gather meaningful data to aid in the assessment of the integrity of

a structure through the use of remotely controlled aerial vehicles constructed from mostly generic, COTS components. The vehicles should be capable of carrying camera-imaging equipment to areas requiring observation. Such an aerial vehicle could be used in cases where standard equipment is either not available or unsuitable due to: poor sightlines; height; potentially failing structure; or, other unforeseen and unplanned scenarios.

\subsection{Constraints}

Due to the large variety of materials, exterior and interior considerations, locations, and usages of various structure types, the scope of this study will be limited to the exterior, superficial examination of structures only. Specifically, the focus is on the survey of structures such as bridges, trestles, power line towers, and related fixtures.

For practical and safety reasons the author chose a test site that is located in a low traffic area in San Luis Obispo. Although not generally a hazardous undertaking, any failure during the testing phase of the research project could cause bodily harm and therefore the selection of a remote test site is a prudent precaution. 


\section{Background on Vehicle Types}

\subsection{Helicopters, Non-Coaxial Multi-Rotors, Airplanes, or Lighter-Than-Air Craft?}

There are four vehicle types that could be suitable for the task being proposed: helicopters; non-coaxial multi-rotors; airplanes; and, lighter-than-air craft. Each has limitations and liabilities, but at the same time each can carry the required payload to accomplish the mission. The challenge is to find a solution that will fit the list of constraints:

- Low cost

- Maneuverability

- Small size

- Relatively low weight

- Ease of use

- Maintainability

\subsection{Low Cost, Inexpensive Construction}

The most important guiding principle of this thesis is to make the craft inexpensive, but not cheap; the distinction being that the selected vehicle type will need to be a high precision machine capable of reuse and long-lasting durability. The chosen vehicle must be sturdy and small enough to be shuttled from location to location, while at the same time simple enough to be deployable in a matter of minutes. The cost of the vehicles must be low relative to turnkey 
systems, while offering similar performance and reusability. This limits the cost of a fully operational system to approximately (US) $\$ 3000$ (2010).

\subsection{Maneuverability}

A primary consideration for the craft is that it be maneuverable in all directions, while being able to hold a static position and a given heading, yet stable enough to carry precision equipment and fly long enough to make meaningful survey observations. It should be capable of moving from one location to another with relative ease while allowing for controlled flight around any obstructions that may exist.

\subsection{Size and Weight}

The overall mass of the craft while not in operation and with the included imaging equipment, should fall between one and a half and six and a half kilos. It should be a mass low enough such that the average person can handle it without too much difficulty. It should fit in a regular vehicle or minivan, with the goal being that it is easily transportable. The size, while small, should not be so diminutive that it cannot carry the required equipment. Lastly, it should be large enough so that it can operate outdoors in winds up to 16 kilometers per hour (i.e., 4.5 meters per second), and small enough so that it can operate in close quarters without creating dangerous turbulence to the structure around which it is operating. 


\subsection{Ease of Use and Performance}

Operation should be straightforward and simple enough that a novice without significant flight experience could operate it after a brief tutorial. Flight control augmentation equipment should be employed where possible so that the operator(s) can concentrate more on data gathering, and less on the mechanics of flying. The performance envelope should allow that the vehicle can carry the required equipment and still be flying within its normal operating parameters.

\subsection{Maintainability}

Maintenance must be straightforward and simple enough so that basic field repairs can be undertaken on the spot without the need for specialized tools, thereby ensuring maximum operational time. The objective of using COTS equipment is that parts are readily available. Use of COTS hardware means that major repairs and maintenance can be performed relatively quickly, by replacing parts rather than having to manually fabricate new ones.

\subsection{Advantages and Disadvantages of each vehicle type}

\subsubsection{Helicopters}

Helicopters, both remote controlled models and full-scale versions, are mature technologies. The design and mechanics have evolved over the past 86 years into the large selection of sizes and configurations available today. Figure 2-1 is an example of a modern remote controlled model helicopter. This example is electrically powered, hence the visible wiring snaking around the outside and lack of an exhaust pipe and exhaust gasses. 


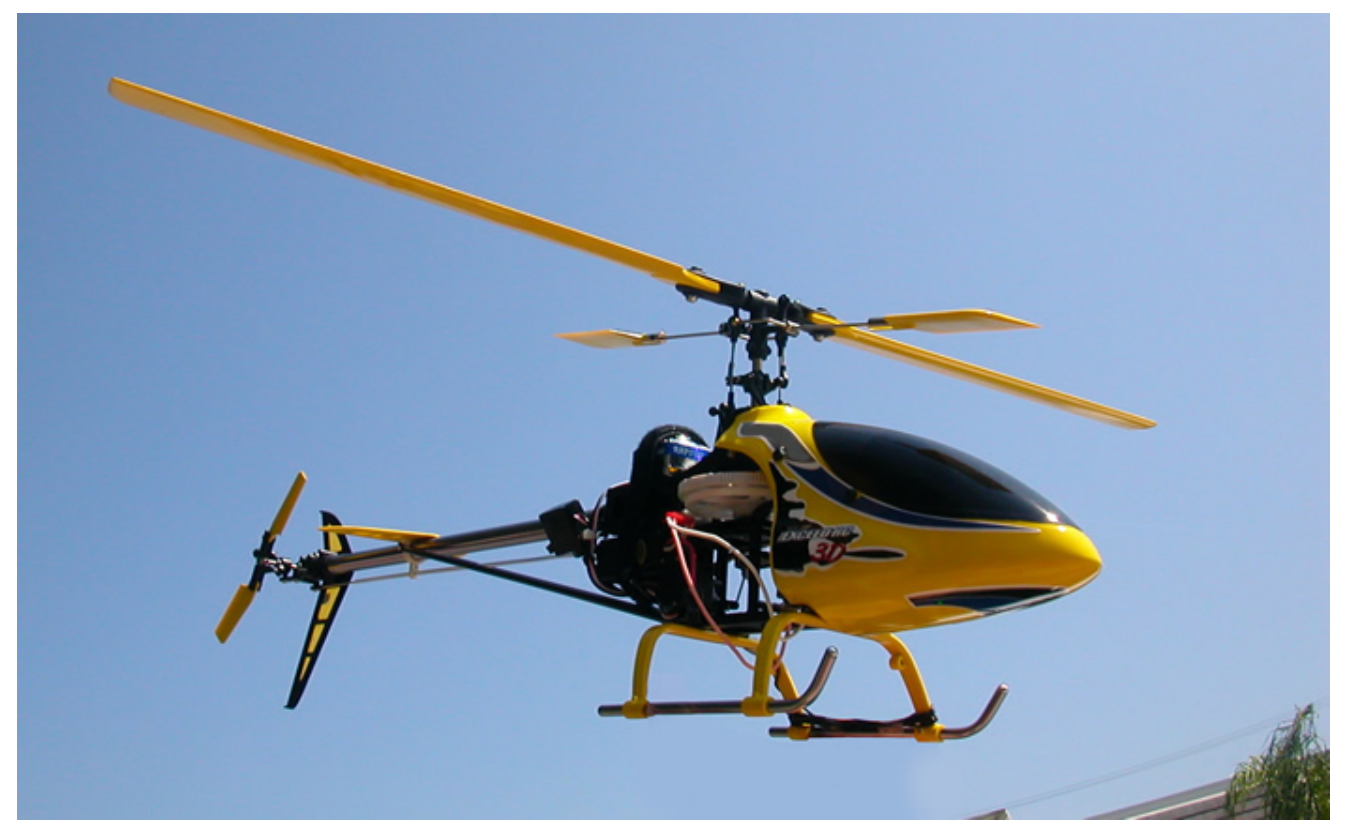

Figure 2-1, Typical model helicopter in flight. (c) http://www.nitroplanes.com/rag26ccrarec.html

Helicopters enjoy a relatively wide agility envelope. Operation in three dimensions coupled with the ability to remain static in the air differentiates them from many other flying aircraft. Remote controlled helicopters enjoy a level of agility unseen by their full-scale counterparts, such as inverted flying and near instantaneous changes in direction. However, this performance comes at the price of limited range and limited load-carrying capacity. Up to $20 \%$ of a helicopter's power is used to counter the torque produced by the lifting rotor (Leishman 2006). From a general point of view this means that a helicopter must be relatively overpowered in respect to its weight. The control linkage setup at the rotor head is a complex collection of hardware, the most important element being the swash plate. The swash plate is responsible for controlling blade pitch and subsequent helicopter direction from statically mounted input actuators. 
Figure 2-2 illustrates the swash plate from a small electric helicopter. (Courtesy of HeliMax ${ }^{\mathrm{TM}}$ helicopters)

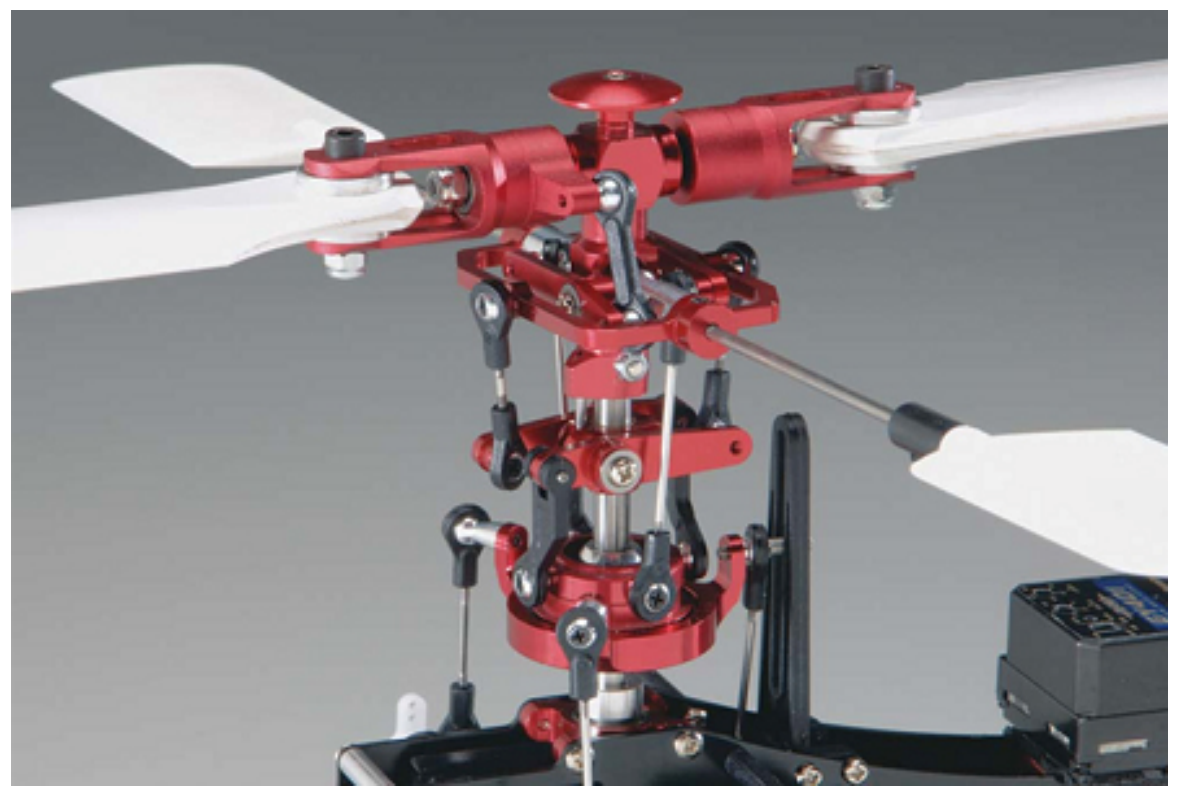

Figure 2-2 This is an example of the swash plate and rotor head control linkages.

It is not uncommon to see model helicopters at the model fields flying long after the conditions have become too windy for model airplanes. Helicopters are far less sensitive to the effects of wind on their flight behavior. Remote controlled helicopters, without the need to protect a pilot can do everything a full-scale helicopter can accomplish, but with more radical maneuvers. It is not uncommon to see model helicopters flying inverted, or even flipping end-over-end within a few meters of the ground. These types of maneuvers are not currently possible in full-scale helicopters.

In the domain of remote controlled equipment, helicopters are available from a vast number of manufacturers, with almost unlimited sizes and prices to choose 
from. Helicopters can be found in electric, gasoline, nitromethane and kerosene powered variants. Each of the variously powered models has its own advantages and disadvantages in relation to flight characteristics, maintainability, usability and complexity.

Finally, the ubiquity of model helicopters has led to cottage industries that offer an almost unlimited number of upgrade parts to specialize and customize the machines, all off-the-shelf. These upgrades are often used to increase the performance envelope, and more often than not are geared toward the flyers that do the most extreme stunts and maneuvers, where high strength and rigidity are critical.

\subsubsection{Non-Coaxial Multi-Rotors}

The typical non-coaxial multi-rotor machine uses between three and eight motor/propeller power pods. Each motor is directly coupled to a propeller, and the thrust of each propeller is directly represented by the rotational speed of the motor. Each motor is fed power by a central processor that synchronizes it with the other motors. The processor then takes input from the operator, translates it to intent, and moves the machine accordingly. In flight they are very stable, and can maneuver and rotate in almost any direction. Figure 2-2 represents one version of a non-coaxial multi-rotor. Some versions have three motors; some have as many as eight. 


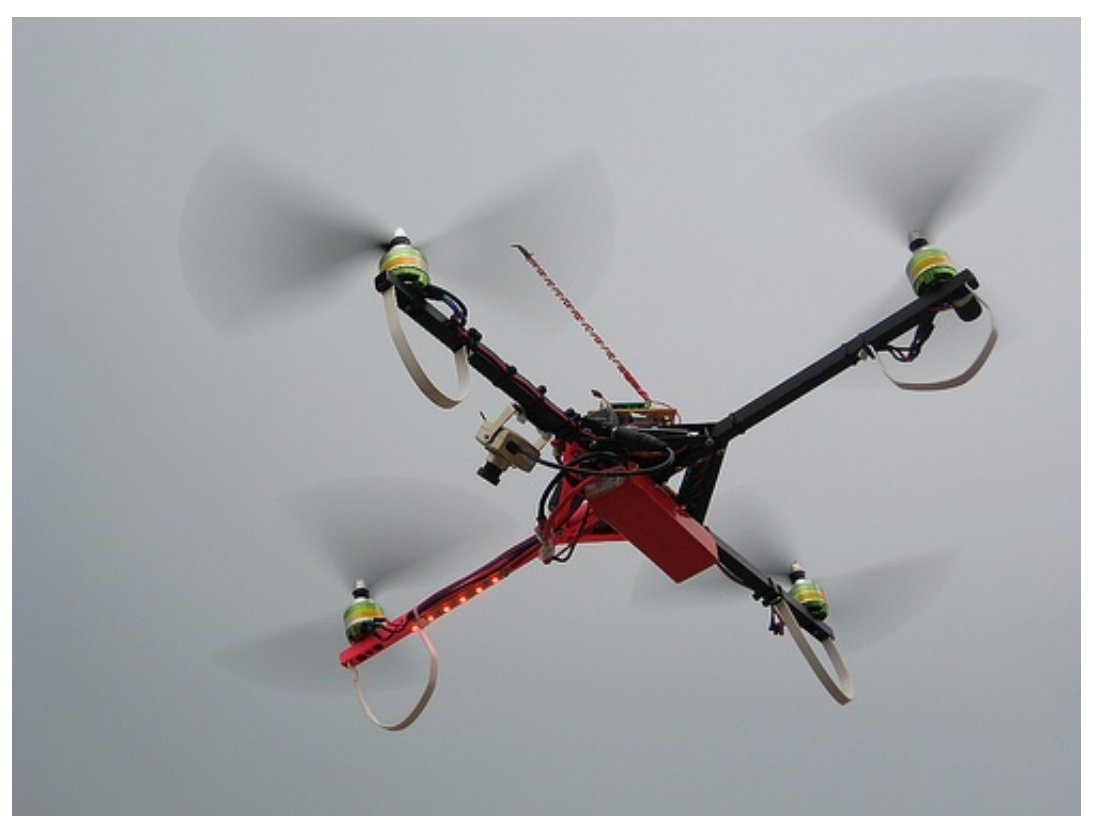

Figure 2-3 Quadcopter in flight, one of many variants of non-coaxial multi-rotor aircraft. (C 2009, Stevens Institute of Technology

Although the multi-rotor concept has been around for a number of years, it has only been in the last four or five years that they have become, more or less, a reality in modeling. The forward leaps in technology, both in battery power and solid-state miniature gyroscopes and control systems, have allowed multi-rotors airframes to be flyable and controllable. A multi-rotor can do everything a helicopter can do, but since it has multiple propellers spinning in opposite directions there is no torque element that needs to be cancelled by a vertically placed fan, as in a helicopter, so all the power can be used for vertical lifting.

The negative, of course, is that there are multiple motors, not one. Each motor has to have a corresponding speed controller. Even with the perceived redundancy, if any one motor or related control unit fails the craft will be rendered almost inoperative. There are some ultra high-end models that have two motors 
and speed controllers for each pod and will continue to fly with any singular, or perhaps multiple failures, but those fall outside the scope of this project, primarily due to the very high cost of this type of specialized equipment.

\subsubsection{Planes}

Model planes are straightforward and relatively simple to fly, compared to a helicopter. Almost Ready to Fly (ARF) models are widely available for under $\$ 100$. They require only modest skill to build, and come in a large array of shapes and sizes. The main advantage of airplanes over helicopters is flight duration. Flight times can exceed 40 minutes with a plane, whereas helicopters average only ten minutes. Airplanes can carry heavy loads and are very stable camera platforms. If electrically powered, the motor can be turned off during photography to eliminate vibration, and turned on again when required. The desired configuration is such that the imaging equipment is located away from the motor and spinning propeller, but still protected in the event of a rough landing. Figure 2-3 illustrates one of the model kits that is widely used as a camera carrying vehicle. Multiplex's EasyStar is light-weight, simple, rugged, and can be assembled in just a few hours. 


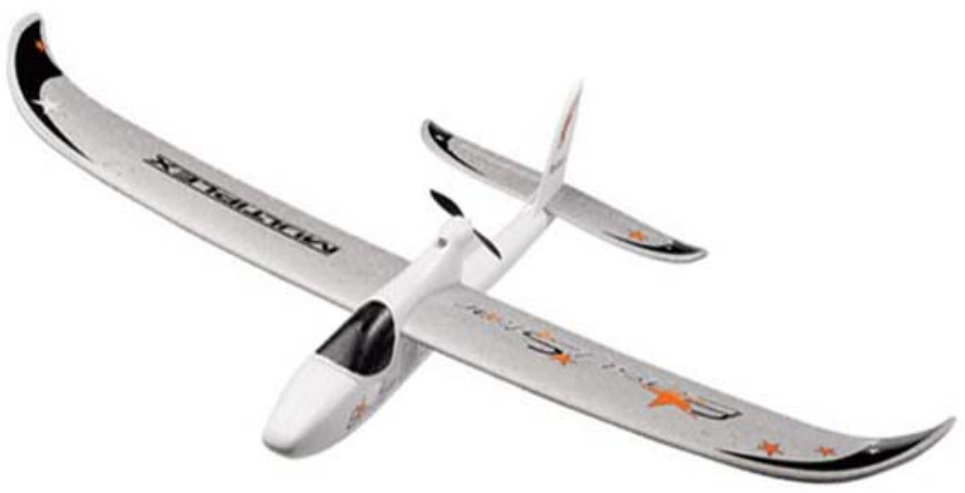

Figure 2-4 Multiplex EasyStar, an inexpensive, easily modifiable model plane

Like helicopters, if a plane has an engine failure it is capable of gliding to a landing. However, unlike Vertical Take-Off and Landing (VTOL) vehicles, airplanes generally require a prepared space to both land and take off. Some planes, like the one in Figure 2-3 can be launched manually with a fast hand movement under full power, but they are generally not caught during landing. Furthermore, the more weight a specific airframe carries, the longer the required runway, thereby increasing the space requirements. Many of the turnkey vehicles use a launcher and arresting landing net, but that increases the cost, equipment and manpower.

The major drawback of the airplane in the scope of this project is its inability to hold a static position. The requirements of being able to take close range still pictures in challenging environments do not suit the airplane. While there are variants that can hover like a helicopter, and fly like a plane, these are more 
complex machines that do not have the carrying capacity and maneuverability to fulfill the tight-quarters flying required for this project.

\subsubsection{Lighter-Than-Air Craft}

Lighter-than-air vehicles, commonly called blimps or aerostats, use helium as a lifting gas. Helium has a tendency to leak out of the gas envelopes of the blimps or aerostats and must therefore be replenished from day to day. An aerostat's envelope or gas sack is not rigid like that of a zeppelin, so when the gas is removed, the entire structure collapses like an empty balloon. The benefit of this is that they can be packed into a relatively small package. However, the envelope materials are quite delicate, and therefore must be treated with care to avoid being punctured or torn.

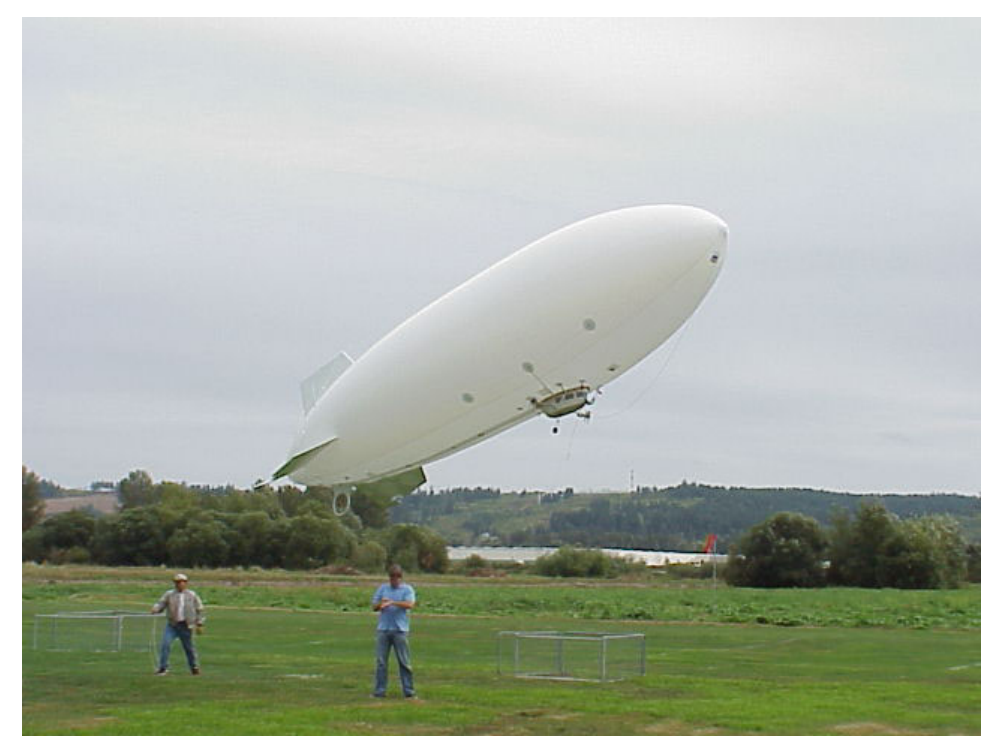

Figure 2-5 A payload toting blimp with belly camera. @ Ryan Winslow, www.flypau.com

Lighter-than-air vehicles offer the most stability of the vehicles in this comparison, but require the most setup time and have the most limited flight envelope in all but the calmest environments. Aerostat size increases in direct relationship to 
the payload. In Figure 2-4 it is possible to see the gondola that is suspended below the gas envelope. The disparity in the size of the gondola versus the gas envelope is one of the weaknesses of using lighter-than-air vehicles for observation in the context of this thesis. Since they have relatively large side and front profiles, they are slow moving and susceptible to wind forces. They are also difficult to transport, since they can be quite large, on the order of four to nine meters in length, to gain enough lift to carry simple telemetry and camera equipment. They must operate in relatively calm conditions to avoid the risk of crashing. With the proper equipment it is possible to deflate and recapture the helium, but that is time consuming, and the recapturing equipment is expensive.

\subsection{Vehicle Choice}

After having weighed the benefits and shortcomings of each vehicle type, this researcher felt that the helicopter would be the best choice. The helicopter airframe satisfies most of the criteria including:

- Low cost

- Simplicity

- Ease of transport

- High parts availability

- Proven design

Further analysis of the helicopter's strengths, weaknesses and variations will be explored in Chapter 3 of this thesis. 


\section{Helicopters}

\subsection{Operational Characteristics of a Helicopter}

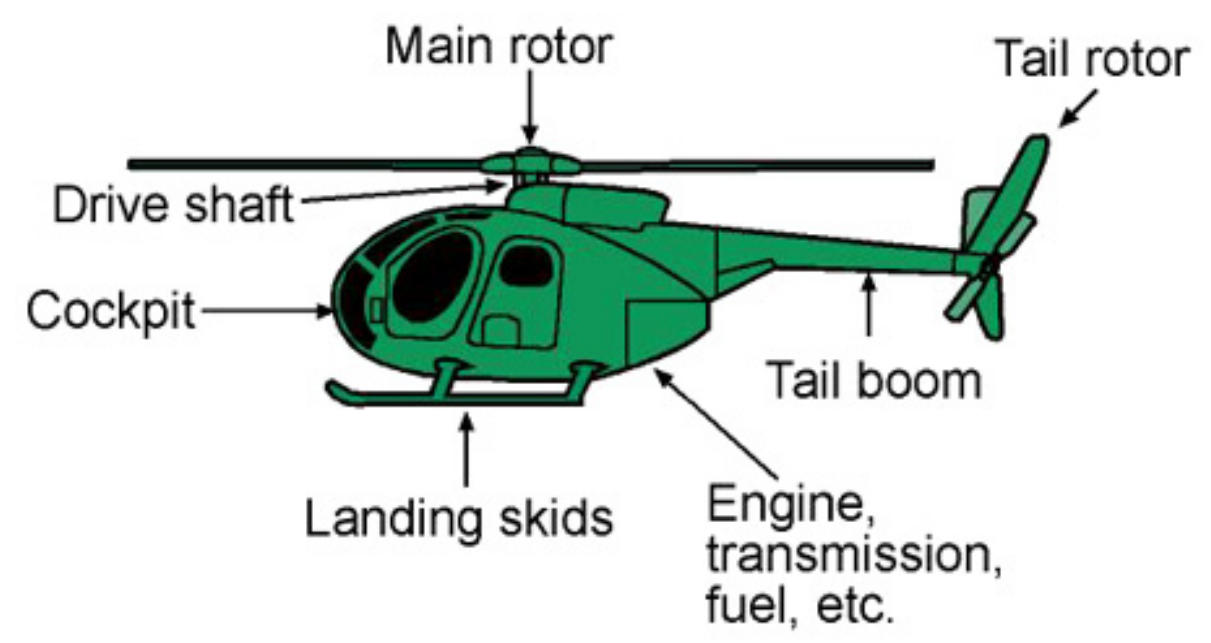

Figure 3-1, Fundamental parts of a helicopter.

A helicopter is a relatively unique flying machine. Helicopters are capable of translating engine power into stable flight in any direction. A helicopter can hover, fly forward and backwards, rotate in place, or perform a combination of these simultaneously. Helicopters can fly in still air or wind, day or night, and in wet or dry conditions. Over the years helicopters have been adapted to operate in many interesting and unique ways, modified to perform delicate tasks or carry heavy loads, to save lives or take lives.

Figure 3-1 graphically portrays the layout of a generic helicopter. While sometimes there are variations to the theme, the basic layout remains the same. In simple terms, a helicopter operates by taking the rotational power produced by its engine(s) and splitting it, via a transmission, into rotational energy in two 
planes. The first plane is horizontal for the main rotor; the second is vertical, the tail rotor. Due to parasitic and induced drag in the main rotor blades as they spin, the helicopter is influenced to twist in opposition to the rotational direction of the main rotor. In order to counteract this twisting a smaller rotor is fitted at the rearward end of the fuselage that pushes against the generated torque of the main rotor drag, canceling it. This resistance to the torque is what allows the helicopter to hold a particular heading. In order to yaw ${ }^{1}$ the helicopter while hovering, for example, the tail rotor either pushes harder against the generated main blade torque, or pushes less against the torque thereby allowing the fuselage to rotate. Modern full-scale helicopters and model helicopters, can be fitted with systems that incorporate gyroscopes to monitor the heading angle of the helicopter, and counter it automatically so that the helicopter can maintain a set heading. In this way it is possible to reduce pilot workload, as well as keep the aircraft pointed in the desired heading despite wind gusts or other forces that might be acting on it.

In the event of a power failure a skilled pilot can land a helicopter, full-size or scale, almost as softly as if it were flying under full power. With a model helicopter autorotation is achieved by converting a powered main rotor into a freewheeling windmill by reversing the pitch of the blades. The falling helicopter is able to convert the potential energy of altitude into rotational energy in the main blades. This is then reversed as the helicopter approaches the ground.

\footnotetext{
1 Yaw refers to a change in angular heading. In the case of helicopters, yaw describes rotating left or right during forward or backwards flight, or while hovering.
} 
The wind milling blades are once again reversed, their pitch transitioned into normal flight configuration. This slows the helicopter, and allows it to fly as a powered helicopter until the potential energy is exhausted. However, a poorly executed autorotation will lead to a crash (Messiter, 2007).

Flight performance of a helicopter comes at the cost of fuel economy. In this thesis the helicopter is employed as a static platform while hovering, the least efficient phase of helicopter flight. Helicopters are generally more fuel efficient in forward flight, as the rotor disk benefits from transitional lift and therefore less pitch needs to be applied to the blades, requiring less horsepower (Johnson, 1994). The benefits of simply adding more fuel to increase flight time is not quite as simple as it might be in a traditional aircraft. As the weight of the vehicle increases, more collective ${ }^{2}$ must be applied to lift it. As a result there is more induced drag, which then requires more power to overcome. So, by adding more fuel the realized benefits are not linear, and the extra fuel will displace other equipment or passengers (FAA 2007).

As with all machines, helicopters have some pitfalls and issues to be aware of. These sensitive characteristics are found in both full scale and model helicopters. Some of these issues arise in high-speed flight, and others in low-speed flight. The low-speed flight issues are the ones that are relevant to this project, as most

\footnotetext{
${ }^{2}$ Collective control, when applied, changes the pitch of all the blades equally. This allows for assent or descent vertically. Alternately, cyclic control allows the pitch of one blade at a time as it rotates around the main shaft. This facilitates movement in the horizontal plane by tilting the rotor disk. Simply put, the helicopter then goes in the direction of the thrust vector from the main rotor disk.
} 
survey work is performed at slow speed or under hover conditions. Some of the issues include, but are not limited to vortex-ring state and pitch up (Padfield, 2007).

The vortex-ring state is a condition that occurs when a helicopter descends too rapidly. Principally, it happens when a rotorcraft descends straight down, or relatively straight down ${ }^{3}$, and is influenced by its own vortex rings. Generally, when flying with forward speed, those vortexes are left in the wake of the vehicle. However, when descending in still air, the vortices at the tips of the blades are pulled back into the thrust stream, which effectively reduces the available lift (Padfield, 2007). Figure 3-2 illustrates how a toroidal ring of air forms around the outer circumference of the main rotor blades, robbing the rotor of lift.

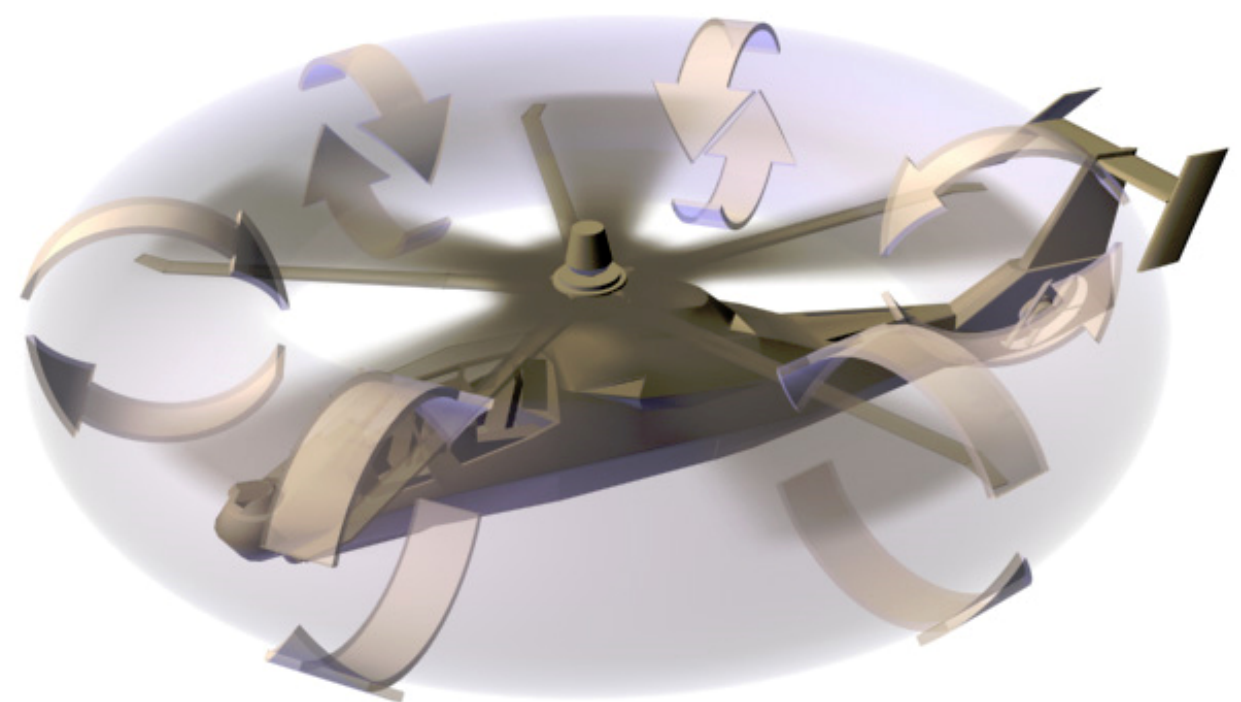

Figure 3-2 This graphic illustrates the toroidal nature of the vortex ring around the outer edge of the main rotor.

\footnotetext{
${ }^{3}$ If the helicopter were to be descending down-wind at the same speed as the wind is blowing, then that could constitute zero airspeed, setting the stage for the vortex ring state condition.
} 
The vortex ring state is also referred to as "power settling". This term has been applied due to the automatic response of many pilots to apply more power and collective pitch to overcome the vortex ring state. However, without sufficient power, it is the wrong approach and will exacerbate the problem. Often the additional parasitic drag of increased cyclic input on the rotor head will totally overcome the available horsepower resulting in loosing rotor speed, or stall the blades altogether due to laminar separation. A second set of vortex rings occurring at the rotor head effectively robs the helicopter of the control that occurs at the innermost section of the rotors. The best solution to save the helicopter from this condition is to reduce collective, allowing the helicopter to fall at a faster rate, and then increase forward velocity by pitching the nose down in order to leave the vortex rings behind the helicopter. Only then can collective be added again, and normal flight resumed. Clearly, if this occurs near the ground the loss of lift could result in a catastrophic crash landing. The vortex ring has been observed in both full scale and model helicopters. The author has experienced power settling with a heavily loaded camera helicopter, but to date has been able to fly out of the condition.

The pitch up condition occurs when a helicopter looses lift in the main rotor due to a stalling of the rotor blades. Specifically, stalling the back of the rotor disk. Mostly this happens when the helicopter is moving at high forward speeds, or high relative airspeed. It is further aggravated when the vehicle is heavily loaded, or there is high disk loading due to a generally large gross vehicle 
weight. If pitch up is allowed to develop without input from the pilot, the helicopter could be placed in a state of total loss of control. The solution is similar to that of the vortex ring, being that the pilot must leave the flight condition that caused the problem in the first place. Often this requires applying control input that is counter-intuitive (Croucher, 2007). When a pitch up condition occurs it is important to slow the helicopter so that the retreating blades are, once again, able to generate lift. Additionally, collective pitch must also be reduced to not aggravate the blade stall condition.

\subsection{Variants of Model Helicopters}

Model helicopters can be surprisingly similar to their full sized counterparts. Without the need to carry humans, and because of their smaller size and the relative strength of materials at these smaller sizes, they can carry much higher useable loads and fly much more aggressively.

Generally speaking there are accepted methods for sizing helicopters based on the types of engines that power them. Traditionally these engines were of the nitromethane type, 5 cubic centimeters ${ }^{4}, 8.19$ cubic centimeters, and 14.74 cubic centimeters in size. A more modern sizing method has gradually emerged based on the length of a main rotor blade, and is power source agnostic. So, typically

\footnotetext{
4 Traditionally these are simply called "thirty size", "fifty size" or "ninety size" engines (based on the American measurement units equivalent of $0.30,0.50$ and 0.90 cubic inches) as a way of describing the general form factor of those helicopters. Blade length, fuselage length and even power may vary from model to model within those designations.
} 
today, one would be flying a $450,500,600,700$ or other size helicopter ${ }^{5}$. There are also subgroups; mini and micro helicopters, some small enough to fit in the palm of a hand, yet sophisticated to the point of operating and flying similarly to full scale helicopters.

Based on the power system and the size and variety of the particular helicopter chosen, the cargo capacity is defined. If the helicopter is small and light, it will be very maneuverable, but it will not be capable of carrying the weight of the camera and transmitters for a suitable duration. On the other hand, if the helicopter is large and highly powered it will generate more noise and potentially threaten the structures that are being surveyed.

\subsection{Power Systems}

It was only 10 years ago that the standard power system for model helicopters was nitromethane. Nitromethane engines are very powerful and produce horsepower and torque suitable to power large vehicles. Most nitromethane engines used in helicopters are two-stroke and operate at very high rotations per minute $(\mathrm{RPM})^{6}$ in order to produce maximum power. Full-scale two-stroke engines as found on motorcycles and snowmobiles are traditionally very loud, and this trait carries over to model engines as well. They can be operated time after time, with rapid refueling. They require external starters and battery packs

\footnotetext{
5 This sizing scheme is based on the length of one rotor blade, and similar to the displacement description mentioned above, it is applied to the description of the general category of helicopter, and not necessarily a specific vehicle.

${ }^{6}$ Often these nitromethane engines rotate at greater than 20,000 RPM, some as high as 30,000 RPM.
} 
to run those packs and to heat the glow plugs. High percentage nitromethane mixtures can cost upwards of (US) \$6.50 (2010) a liter.

In larger helicopters two-stroke traditional gasoline engines are also used. Gasoline powered helicopters can carry heavy loads. Gasoline motors have the simplicity of pull starters and sparkplugs, requiring much less ground equipment. They do, however, vibrate as much as nitromethane engines. Gasoline is relatively inexpensive but the gas model motors can be quite expensive.

With advances in battery technology, first nickel cadmium (NiCd), then nickel metal hydride $(\mathrm{NiMH})$ and most recently lithium ion phosphate (LiPo) battery power has become, in many ways, the most prevalent power source for small to medium-sized helicopters. Generally speaking, battery power is very safe, and stable. Batteries can be stored fully charged and are ready when required. Some of the newer batteries can be charged at very high rates, and can discharge hundreds of amps instantly if the application warrants it.

Each of the three types of batteries listed above have different properties that make them more or less suited for model helicopters. NiCd batteries, while comparatively inexpensive, are heavy in relation to their power density. They can discharge at very high rates without damage, but due to their weight yield very short flight times. These batteries are rarely used any more in helicopters. $\mathrm{NiMH}$ batteries have a much higher energy density to weight than NiCd batteries. They have the ability to discharge at high rates, and they can be recharged at 
high rates. They do not have a "memory", and therefore their lifespan is much longer than NiCd batteries. These are still used in helicopters, but like NiCd batteries, they yield relatively short flight times. LiPo batteries are the current cutting edge in non-experimental off-the-shelf batteries for model enthusiasts. They are stable in storage, but require care when charging, and are therefore best charged in fire safe environments. LiPo batteries can remain charged for long periods with very low self-discharge. LiPo batteries do have the unpopular distinction of spontaneous combustion when short-circuited, charged too quickly, punctured, or otherwise damaged. Each of the battery types requires special recharging protocols, and therefore special chargers.

Electric motors are the accompanying piece to the battery. They convert the stored energy to mechanical work. The preferred motors for electrically powered helicopters are brushless, running on direct current and controlled by sophisticated speed controllers. The brushless speed controller is capable of high switching frequencies that, in turn, regulate the speed of the motor by sensing and maintaining the motor's speed in revolutions per minute (RPM).

There are two primary variations of brushless motors applicable to model helicopters, the inrunner and the outrunner. The inrunner carries the magnets on the rotor and can spin at high $\mathrm{Kv}^{7}$, but generally with low torque. These are well suited to motors that are highly geared so that the benefits of high RPM can be

\footnotetext{
$7 \mathrm{Kv}$ is the RPM achievable at one volt, and varies from motor design to motor design. $\mathrm{K}$ is a constant of rotation, multiplied with the $\mathrm{v}$, or voltage being applied. For example, a motor with a $\mathrm{Kv}=1000$, when plugged into a 12 volt battery would yield 12,000 RPM at the motor shaft.
} 
realized. The outrunner, on the other hand, carries the motor's magnets on the exterior casing and is the element that rotates, the stators do not move. Outrunning motors, except in some very specific cases, have a significantly lower Kv rating than in-running motors, but they produce considerably more torque. The increased torque often eliminates the need for gearboxes ${ }^{8}$.

Electric power systems have no warm-up time prior to the application of full power, nor do they induce the large vibrations and exhaust gases inherent with internal combustion power sources. A battery's weight does not change during its discharge cycle, and therefore the center of gravity of the aircraft does not change. Time between flights depends on the rate of battery recharge, but can be several hours. This necessitates the purchase of several battery packs if back-to-back flights are required. The initial cost of LiPo batteries (the best combination due to a favorable energy density/weight ratio) is quite high. If charged and used according to the manufacturer's specification a battery can be recharged 300 or more times, which offsets the initial cost and makes it equal, or less expensive than nitromethane fueled engines.

The last and currently most exotic power method is kerosene powered turbine (jet) engines. These are very powerful due to their high RPMs and are virtually vibration free. Jet engines are very loud and can pose a fire risk as they operate at very high temperatures. The high initial costs relative to other methods of

\footnotetext{
8 The elimination of the gearbox is mostly found in model airplanes, although at this time the Muliplex Funcopter, is sold as a gearbox-less outrunner powered model helicopter.
} 
propulsion, complexity of the system, and extensive maintenance cycle make turbines impractical for this application.

\subsection{Power Choice}

To meet the requirements and constraints that were defined for this thesis it was important to find a combination that is: quiet; least expensive; simple to maintain; safe; and, functionally vibration free. The effort to reduce vibration would be very challenging for an internal combustion engine. Exhaust fumes and oily residue from the burnt fuel would interfere with the camera and video equipment. Although smoothly operating, the high cost and noise from jet power effectively eliminates it as a choice.

Ultimately it is the simplicity, economics, smooth operation, and reliability of the electric power system that makes it the most attractive choice. The modular nature of electric power systems allows for a large variety of customization and refactoring. While more expensive initially, electric power systems, over their lifetime, are the most economic option. 


\section{Selecting and Building the Aerial Platform}

\subsection{Helicopter Selection}

There are a number of electric helicopters on the market, from various manufacturers in different countries. This thesis requires a helicopter that is lightweight, simple to assemble, easy to maintain, and can accommodate payloads that are heavy relative to the weight of the helicopter.

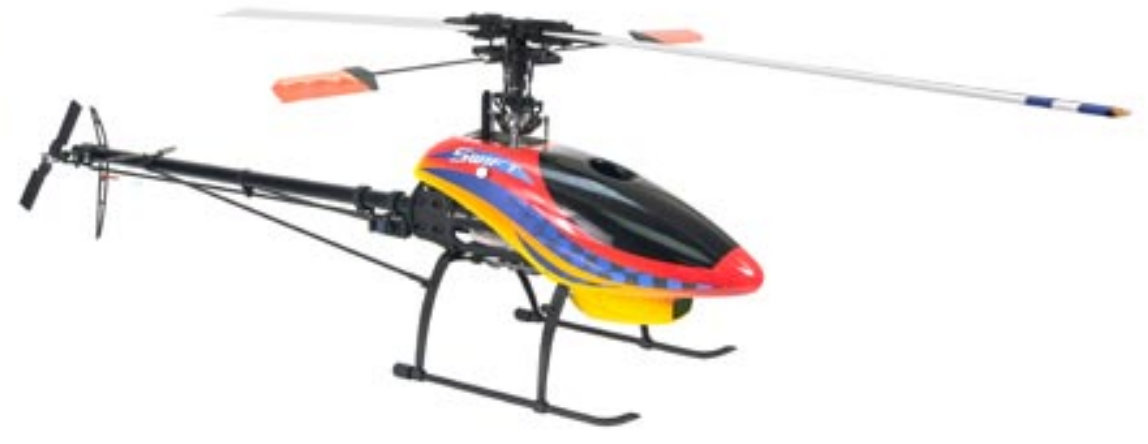

Figure 4-1 Swift 16 by Century Helicopter as it appears when built unmodified. (C) Century Helicopters

After a thorough search, the Swift 16 as pictured in Figure 4-1, by Century Helicopters of San Jose, California was selected. The Swift 16 was a relatively new offering at the time it was selected, but there were hundreds of reports of its quality build, low flight weight, low part count, and reliability. The helicopter frame itself is inexpensive by $30^{9}$ class helicopter standards.

\footnotetext{
9 This is an electric helicopter that was released at about the same time the classification system was changed. The Swift is roughly the size of a 5 cubic centimeter nitromethane powered helicopter. The Swift 16 has a rotor length of 525 millimeters, and would fall into the category of a "500 class" helicopter under the new system.
} 
The Swift 16 kit itself consists of a mostly plastic crutch $^{10}$, with a carbon fiber battery plate and hollow aluminum tail boom. The main motor drives the tail rotor via a fiber rubber belt that twists 90 degrees down the interior length of the tail boom. The tail drive belt is indented and fits into toothed wheels at either end. This is a popular method of driving model helicopter tail rotors due to simplicity, reliability and low wear. The design and layout of the crutch leave ample room for mounting external equipment; - another element that weighed in favor of the selection of this particular kit. There are three separate horizontal surfaces available to accommodate flight control devices and add-on equipment.

\subsection{Power System}

The Swift 16 kit package does not include a motor. After careful consideration the Century 600 outrunner motor was chosen. Although there are literally hundreds of motors to choose from, this is a low cost, high performance motor that has received good user reviews. At maximum voltage it can output up to 1000 watts $^{11}$, which is equivalent to about one and one third horsepower.

\footnotetext{
10 The "crutch" includes the main element of the helicopter that houses the main shaft, landing gear, transmission, and tail attachment assembly. This term is specific to model helicopters, and does not apply to their full-scale equivalents.

11 One horsepower is equal to approximately 746 watts.
} 


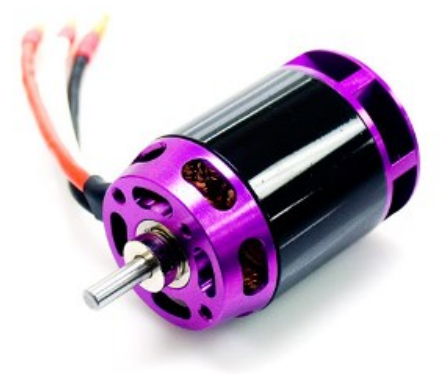

Figure 4-2 Century 600, $715 \mathrm{Kv}$ outrunner with attached cooling rotor.

The Century 600 motor has a $\mathrm{Kv}$ of $715 \mathrm{RPM}$, which is a relatively low $\mathrm{Kv}$ number, but it was considered adequate at the time ${ }^{12}$. Figure 4-2 shows the fixed lower portion and the black rotating upper casing and through-shaft, as well as the rotating cooling turbine at the top. The battery system initially chosen is a five-cell LiPo system. The battery packs were obtained from a vendor named "Cheap Battery Packs", and that turned out to be a bad omen. Each of the two five cell packs that had been ordered ended up with internal short circuits, and was not capable of supplying the amperage it was rated for. This led to the battery packs "puffing"13 as the first step of a run-away chemical process that terminates in spontaneous combustion. Those two battery packs were replaced by a four-cell LiPo battery pack from MaxAmps ${ }^{14}$, Figure 4-3. With a 10 amphour capacity at 14.8 volts and paired with a $715 \mathrm{Kv}$ motor, it yields roughly

\footnotetext{
12 Experience has shown that it is actually lower than ultimately required, and moreover this caused some rethinking regarding battery voltage later due to lower than desired head-speed.

${ }^{13}$ When a pack has a short circuit, internally or via the external plug, the amperage spike will cause internal heat that in turn can cause the pack to expand in size, which is referred to as "puffing".

14 MaxAmps is a battery assembler located in Nine Mile Falls, Washington. They are leaders in the use of LiPo battery technology for model cars, planes and helicopters.
} 
10500 RPM at the motor spindle ${ }^{15}$. The MaxAmps pack can continuously supply

a current of 100 amps, well below what will be required. This power setup proved much more robust, and yielded excellent results.

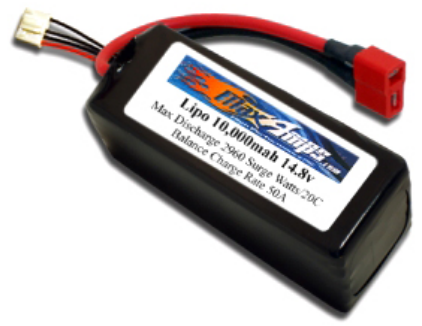

Figure 4-3 MaxAmps 10,000 mAh, 14.8 volt LiPo battery. Weight 820 grams.

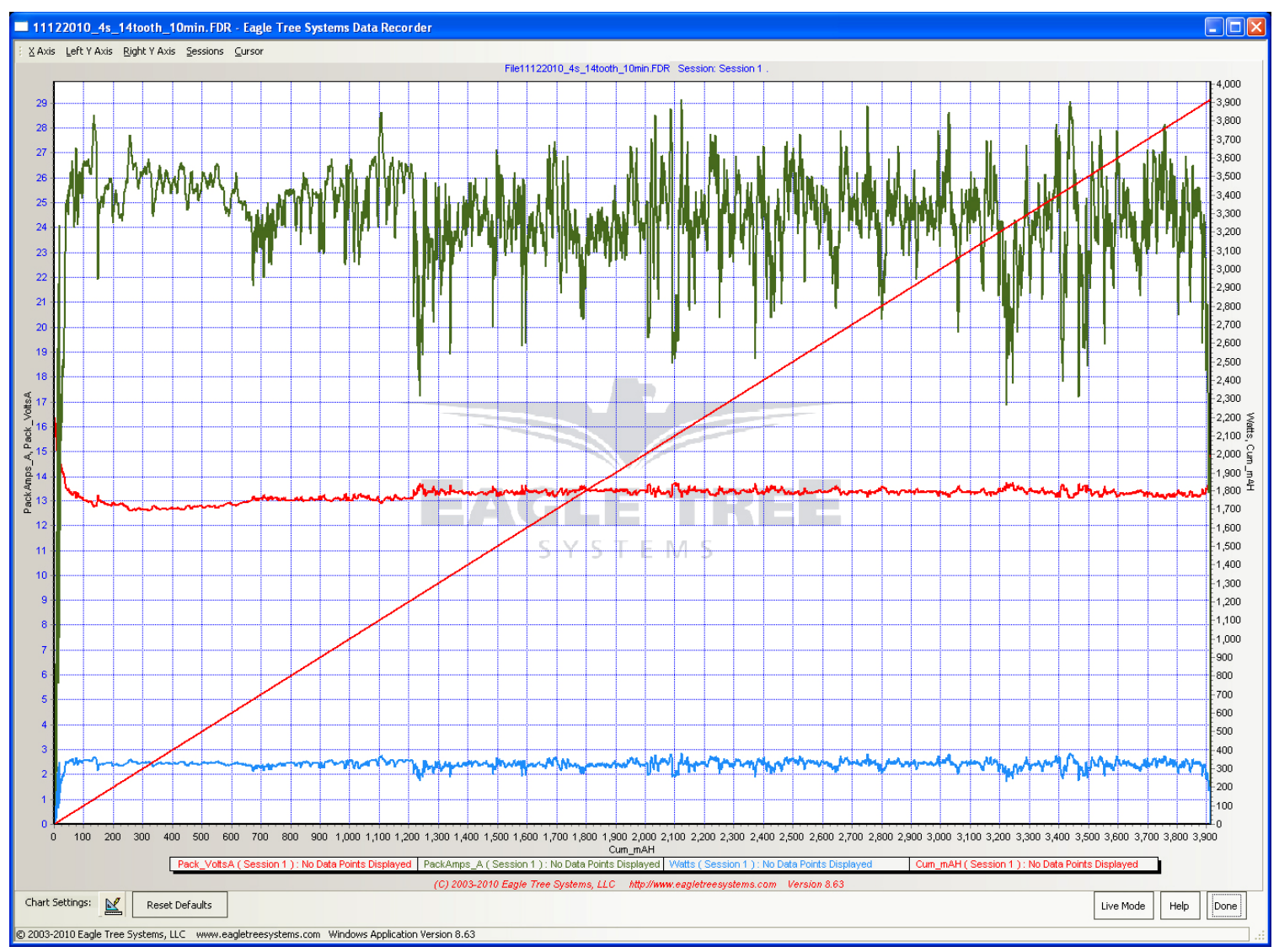

Figure 4-4 Wattage, amperage, cumulative $\mathrm{mAh}$, and battery voltage during a 10 minute flight with a 14.8 volt (four cell), 10,000 mAh battery.

15 The calculation for this is 14.8 (volts) $715(\mathrm{Kv})=10582$. The fully charged battery will have a higher voltage and when almost fully discharged a lower charge. The 10500 RPM number is a good working value to be used as a rule of thumb. 
However, as good as the battery performed in testing, the main rotor speed was not high enough when more equipment was added to the helicopter, and this necessitated a modification to the plan, despite what the initial calculations indicated. Figure 4-4 illustrates that it was delivering roughly 23 amps continuously, roughly one quarter of the battery's rated maximum output. In order to achieve better flight results, another battery was purchased and ultimately became the battery of choice during the rest of the experimentation. This was another LiPo pack from MaxAmps. Figure 4-5, but it was a six-cell pack at 22.2 volts. Figure 4-6 shows that at flight power this battery pack was producing just below 400 watts of power while pulling an average of roughly 16 amps. This yielded roughly 15800 RPM at the motor spindle, and roughly 1850 RPM at the main rotor when efficiency losses were factored into the equation. The most positive finding was that the amperage dropped significantly due to the higher voltage, and this allowed more available overhead when the system was put under a loaded condition.

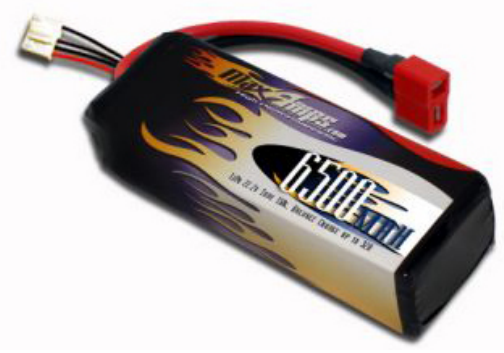

Figure 4-5 MaxAmps 6,500 mAh 22.2 volt LiPo battery. Weight 951 grams. 


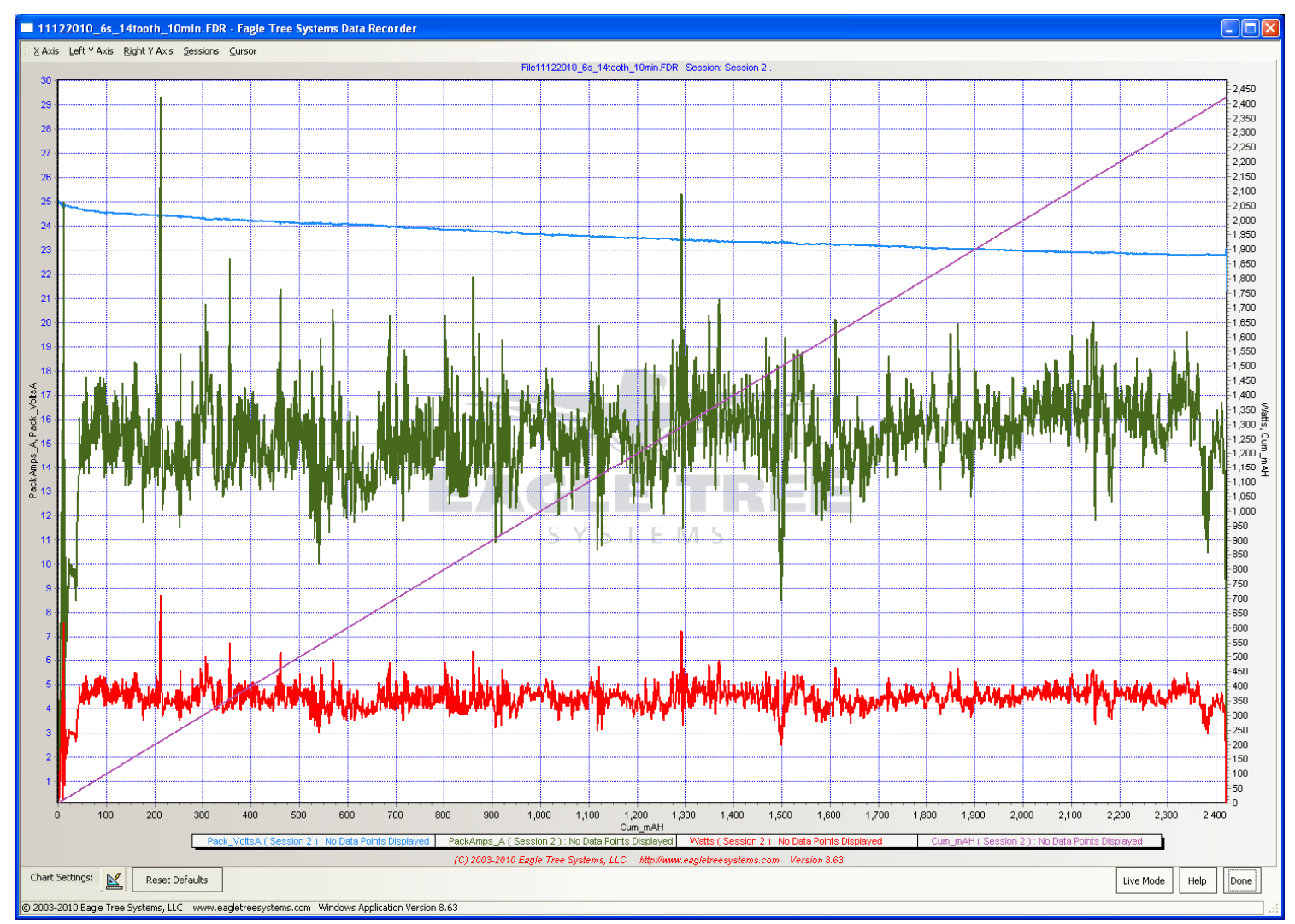

Figure 4-6 Wattage, amperage, cumulative mAh, and battery voltage during a 10 minute flight with a 22.2 volt (six cell), 6500 mAh battery.

Both battery packs yield roughly a 20-minute flight time. The author chose to set a 10 minutes flight alarm, to provide a safety factor in flight time in case of complications in landing, excess wind, or the need to complete further observations.

To control the flow of power from the battery to the brushless motor, a speed controller is required, Figure 4-7. For this project the controller chosen is the Castle Creations "Phoenix HV45" Electronic Speed Control (ESC). Approximate calculations indicate that at a normal hover in still air, with a 14.8 volt battery, the 
current would be on the order of 20 amps (continuously), or alternatively with a 22.2 volt battery about 15 amps. Therefore an ESC capable of more than 45 amps would probably not be required. Furthermore, the "HV" designator on the ESC, indicates that it is capable of high voltage input ${ }^{16}$, and based on research by other model helicopter pilots the $45 \mathrm{HV}$ can safely output up to 60 amps in short bursts with no negative effects.

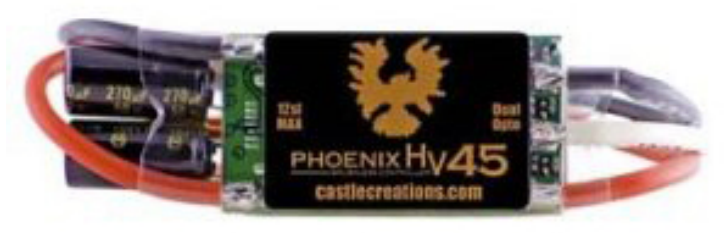

Figure 4-7 Castle Creation "Phoenix HV45 ESC" electronic speed controller.

\subsection{Flight Control System}

In order to operate a remotely controlled vehicle of any kind, there must be means by which the device can be operated from a distance. Airplanes and helicopters share a common type of controller that is generically called a "transmitter". There are hundreds of varieties of transmitters, built by dozens of manufactures. The distinguishing features among transmitters are generally related to their ability to be customized by the pilot. Flexibility to control mixing and programming is the main feature. Additionally, the greater the number of channels ${ }^{17}$ the more sophisticated the radio.

\footnotetext{
${ }^{16}$ Although voltage does not necessarily equate to amperage, this unit was designed more robustly than the "Phoenix 45 ESC", and its durability has been validated by external sources.

${ }^{17}$ A "channel" is generally synonymous to a servo being controlled. A five channel controller, for example, could remotely control up to five servos independently.
} 
In the United States, radio control aircraft have frequencies set aside on the 72 $\mathrm{MHz}$ band, with a unique set of 49 discreet bands. Radio controlled cars and boats operate on higher $75 \mathrm{MHz}$ bands. For both the air and surface frequencies, each transmitter talks to a receiver on a matching band. Should a second transmitter be activated on the same frequency as another transmitter within range of the receiver, the receiver has no way to differentiate which one is actually intending to control it. This leads to a loss of control of the model until one or another of the transmitters is turned off.

In just the last few years the developments of radio transmitter/receiver combinations that operate on the public spectrum of $2.4-2.485 \mathrm{GHz}$ have been developed. These are digital links and are not subject to the same type of interference of their analog predecessors. Each digital transmitter has its own unique identification (ID) that is carried on the data stream. The receiver will only accept packets of data with the correct ID, while all other packets are discarded.

In the most general terms, the radios operating in the $72 \mathrm{MHz}$ bands are interchangeable with receivers in the same bands, despite the brand. The same is not true of those in the $2.4 \mathrm{GHz}$ frequency group, where different manufacturers bind their systems through unique digital strings and protocols. For this reason the researcher chose to operate on the $72 \mathrm{MHz}$ bands.

For this thesis the Multiplex Royal Evo 12 is the transmitter that was chosen, Figure 4-8. The Royal Evo 12 radio can accommodate up to 35 different models 
with individual program characteristics and flight phases. Although more than what was required for basic helicopter flying, the added features of this transmitter allowed for increased pilot safety through redundant throttle cut-offs.

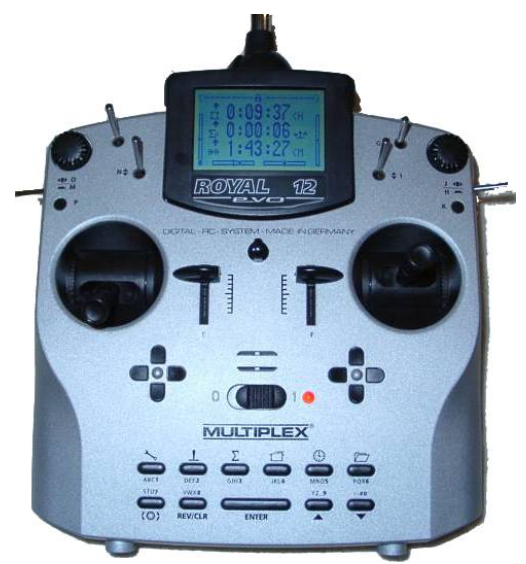

Figure 4-8 Multiplex Royal Evo 12, 12 channel radio transmitter

However, the transmitter is only half the equation. On the helicopter itself is located the receiver. The receiver is the piece of hardware that translates the motions on the transmitter sticks and switches, to mechanical motion of the servos. In this case the receiver is a Hitec Supreme IIS 8 channel receiver ${ }^{18}$, as seen in Figure 4-9. It operates on the $72 \mathrm{mHz}$ frequency. This particular unit utilizes a dual conversion filter ${ }^{19}$, and also automatically detects for positive or negative frequency shifting, a legacy standard employed by various manufacturers to differentiate their own transmitter-receiver products.

\footnotetext{
${ }^{18}$ This particular receiver weighs about 28 grams, and will operate at between four and six volts, typically.

19 "Dual conversion" indicates that it filters all the incoming signals twice, looking for glitches or other signal abnormalities. These transient signals are rejected and therefore not sent to the servos.
} 


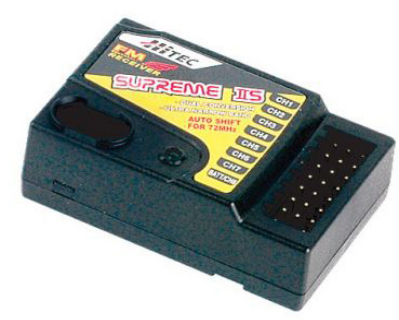

Figure 4-9 Hitec Supreme IIS 8 Channel remote control aircraft receiver

In some situations, the ESC will distribute power to the servos and receiver from the available power of the main battery. The researcher did not want to rely on the power of the main LiPo battery as the loads and power requirements of the drive motor could potentially cause receiver voltage dropout. Instead, a second battery, a JR 2200 mAh NiMH five cell, six-volt receiver battery was added to the system. This second battery will allow the receiver to function with or without the main drive battery. A visual battery status indicator was added to the outside of the crutch, to allow for quick assessment of the state of the receiver battery. A side benefit of using a receiver battery for servo control and receiver control is that bench testing and calibration can be performed without the need for the main drive system to be energized, which is an important safety consideration.

This Swift 16 employs a total of four servos for flight control. Three Futaba S3010's ${ }^{20}$ are in the crutch and are "mixed" 21 via the transmitter and/or other

\footnotetext{
20 The S3101 is a high torque servo capable of $6.5 \mathrm{~kg}-\mathrm{cm}$ of torque at $6 \mathrm{v}$ and can rotate 60 degrees in 0.16 seconds.

${ }^{21}$ Mixing allows servos to be moved proportionally to how they would move when three servos are moving together while being fed four inputs. This facilitates removal of mechanical systems and allows for a more direct servo-to-control surface linkage. Ultimately this reduces slop and failure points in the mechanical system.
} 
flight systems, and one Futaba $9254^{22}$ high-speed servo on the tail boom that provides pitch control to the tail rotor.

The final element in the flight control system revolves around a flight augmentation computer that resides on the helicopter. This system shown in Figure 4-10 is called a HeliCommand $3 \mathrm{~A}$, and through the use of three axis gyroscopes it is capable of maintaining control of the helicopter without any input from the pilot. The HeliCommand $3 \mathrm{~A}$ unit sits logically between the receiver and the servos. There are three modes in which the HeliCommand operates. Stage 0 is off. The system does nothing but allow the signals from the radio transmitter to drive the servos. If the vehicle were to roll off axis, or otherwise leave the stable flight envelope, it would be the pilot's responsibility to regain control. In stage 1 , the three gyros internal to the HeliCommand unit work in concert to maintain a level attitude, as well as hold a particular heading. Any input from the pilot at this point, will be run through the flight computer within the HeliCommand, compared to the data gathered from the gyros, and then interpreted into servo movements to achieve the motion in the commanded direction. The final mode, stage 2, allows an optical sensor built into the HeliCommand system to view the terrain below the helicopter, and input automated control responses to hold a current position. This same system will also judge altitude and mitigate collisions with the ground.

\footnotetext{
22 The $\mathbf{S 9 2 5 4}$ is a high-speed digital servo that can rotate 60 degrees in 0.06 seconds, and is designed for tail rotor control.
} 


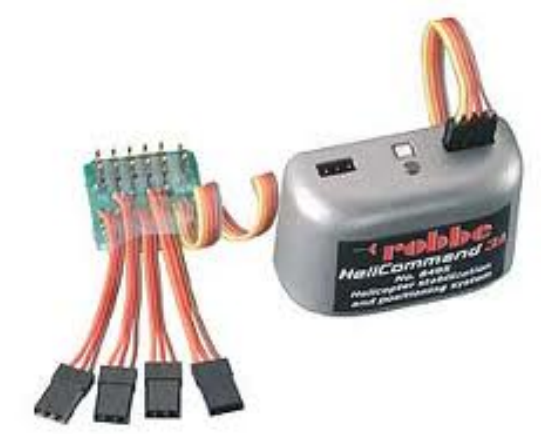

Figure 4-10 HeliCommand 3A Flight Augmentation Computer and Interface.

By utilizing a system, such as the HeliCommand, it is possible to remove some, but not all, of the flight burden from the helicopter operators allowing them instead to concentrate on setting up and positioning the helicopter for photoreconnaissance of the structural elements that they are interested in viewing.

\subsection{Image Telemetry}

The final element in the package is the ground station. This is simply a telemetry base-station that allows the pilot to see video through the lens of the camera, which is mounted on the helicopter. The system consists of four components:

- Camera

- Transmitter

- Receiver

- Video display screen 
The entire telemetry system fits in a single small briefcase for transport. The briefcase includes its own power supply ${ }^{23}$, tripod to mount the antenna, a patch antenna for the $2.4 \mathrm{GHz}$ receptions, the transmitter, a digital camera ${ }^{24}$, and video screen for the real-time helicopter video feed. All the elements in the briefcase are held in place by industrial strength hook and loop connectors. With this method of attachment it is very simple to reconfigure the base station as required.

The video transmission from the helicopter to the base station is achieved with a 200 milliwatt, $2.4 \mathrm{GHz}$ transmitter/receiver from Black Widow AV. Figure 4-11 shows the receiving unit on the left, and the transmitting unit, the one fitted to the vehicle, on the right. This audio/video transmitter has an effective range of More than one kilometer, line-of-sight. It is connected to a standard 4 mega-pixel digital camera from Pentax, an Optio 43WR. The camera uses its own built-in battery for picture taking, and runs on a self-timer that takes one picture every 10 seconds, or if a servomotor is attached to the camera, can be triggered by the pilot or assistant while watching the live video stream on the ground-station monitor.

\footnotetext{
${ }^{23}$ A single 12-volt gel-cell motorcycle battery can power the entire system for up to 5 hours.

${ }^{24}$ The transmitter and the camera are both mounted on the helicopter as stand-alone devices.
} 


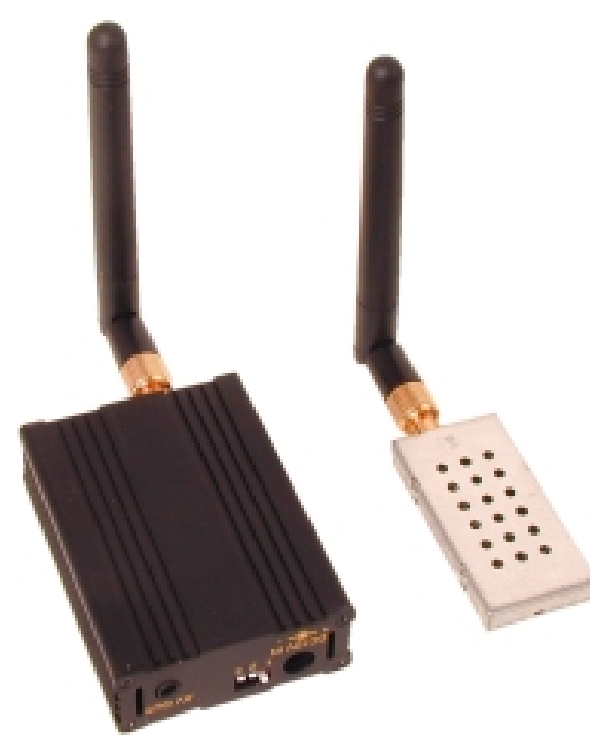

Figure 4-11 Black Widow $2.4 \mathrm{GHz}$ audio/video transmitter and receiver.

\subsection{Final Vehicle}

The helicopter platform has seen a number of revisions in its development, and will probably continue to be updated and upgraded after this thesis is complete. However, at this time it has come to a stable point in development and has a cost of approximately (US-2010) \$2600 (Table 4-1). The final package of helicopter, camera, transmitter, and base-station for the audio and video is shown in Figure 4-12.

It must be stated that this price includes some elements that many model enthusiasts would already have (a radio transmitter, for example), and therefore the cost could be lower. On the other hand, at the time this project was started, 2004, many of the control elements, and batteries were new technology, and therefore the cost was higher than it is currently. Additionally, as more equipment became available on the market, the market forces drove those prices down. 
The pricing can be broken down as shown in Table 4-1.

Table 4-1 Cost of vehicle components as currently built.

\begin{tabular}{|c|c|}
\hline Part Name & Cost in 2010 (US) $\mathbf{\$}^{\mathbf{2 5}}$ \\
\hline Multiplex Royal Evo 12 transmitter & 650 \\
\hline Century Swift 16 helicopter & 250 \\
\hline Heli-Command 3A flight augmentation & 580 \\
\hline LiPo 6 cell 6500 mAh battery & 300 \\
\hline Hitec Supreme IIS 8 receiver & 30 \\
\hline Futaba 3010 Servos (3x) & 90 \\
\hline Futaba 9254 servo(1x) & 100 \\
\hline Phoenix HV45 ESC & 130 \\
\hline Century 600 outrunner motor & 110 \\
\hline 6v Receiver Pack & 40 \\
\hline Pentax Optio 43WR 4 mega pixel camera & 130 \\
\hline Black Widow A/V receiver and transmitter & 150 \\
\hline Briefcase base station w/ tripod and battery & 70 \\
\hline Total & $\mathbf{\$ 2 , 6 3 0}$ \\
\hline
\end{tabular}

Table 4-2 indicates the price difference when using equipment that has been developed or has dropped in price due to higher availability or new manufacturing techniques. It must be pointed out that often the lower priced hardware is not necessarily as high quality, but under normal use should work almost as well as what is currently being used.

\footnotetext{
25 Rounded to the nearest $\$ 5$ (due to variability between vendors these prices cannot be stated absolutely), and excluding tax.
} 
Table 4-2 Estimated Cost using alternative or less expensive hardware.

\begin{tabular}{|l|c|}
\hline \multicolumn{1}{|c|}{ Part Name } & Cost in 2010 (US) \$ $\mathbf{2 6}^{\mathbf{1}}$ \\
\hline $\begin{array}{l}\text { Hitec Aurora 9 Synthesized 72MHz Tx \& } \\
\text { Freedom 9Ch Syn }\end{array}$ & 400 \\
\hline Century Swift 16 helicopter & 250 \\
\hline Flight Mentor flight augmentation system & 140 \\
\hline LiPo 6 cell 6500 mAh battery & 200 \\
\hline $\begin{array}{l}\text { Airtronics 94102Z Standard Heavy Duty } \\
\text { Servo Z (4x) }\end{array}$ & 40 \\
\hline Phoenix HV45 ESC & 90 \\
\hline Century 600 outrunner motor & 100 \\
\hline 6v Receiver Pack & Eliminated ${ }^{27}$ \\
\hline Kodak Easy Shot 14 Mega Pixel digital & 90 \\
\hline Black Widow A/V receiver and transmitter & 75 \\
\hline Briefcase base station w/ tripod and battery & 70 \\
\hline \multicolumn{1}{c|}{ Total } & $\mathbf{\$ 1 , 4 5 5}$ \\
\hline
\end{tabular}

26 The alternate cost is the price for equipment that was not available for purchase at the time this project was initiated, but has since been developed, or for equipment that is less flexible, but has similar functionality.

${ }^{27}$ In a budget system this receiver power could be supplied by the ESC, and therefore this battery could be eliminated. 


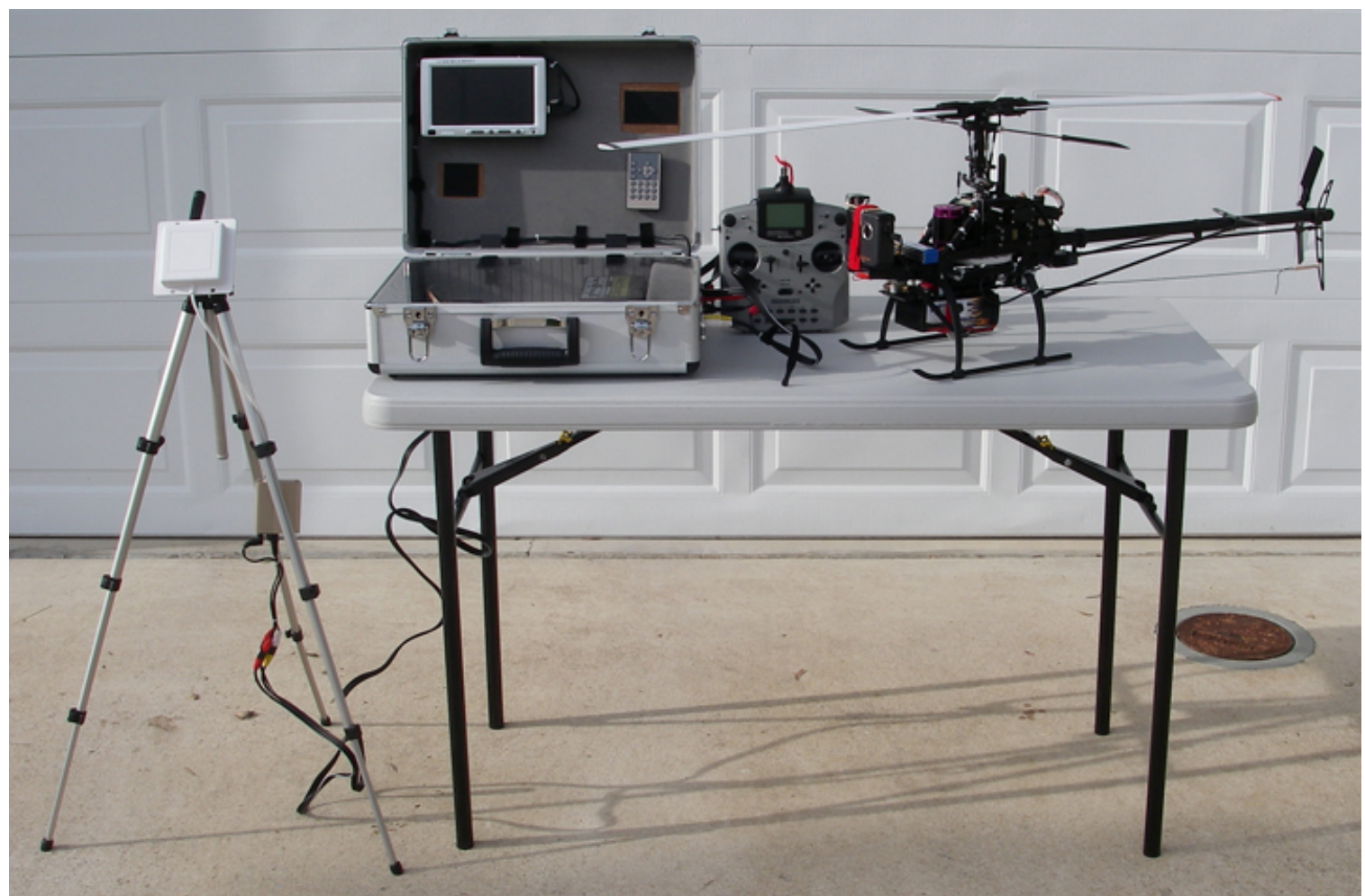

Figure 4-12Century Helicopter, Swift 16 prepped for aerial photography. Included also is the base station, Multiplex transmitter, and the audio/video telemetry base station

\subsection{Helicopter Flight Testing}

In preliminary flight-testing, the helicopter performed very well. Initially there were some issues with main rotor head speed, and those were addressed, first with a higher voltage battery, as explained in Section 3.4, and then later with gearing. As it turned out, the six-cell battery mated with a 14 tooth main gear produced a main rotor speed well over redline. Century Helicopter rates wooden rotor blades to roughly 1800 RPM, maximum ${ }^{28}$. Even without a tachometer it was clear to the researcher through observation that it was an unsafe condition.

\footnotetext{
28 This, of course, does not include some undefined safety margin, but the researcher did not want the risk of one of the rotor blades breaking away from the rotor head with the tip-speed approaching 490 kilometers/hour.
} 
Calculations later showed that if they had been allowed to spool to maximum revolutions, the rotor speed would have been over 2300 RPM.

The main gear was reduced to a 12-tooth gear that produced one good flight ${ }^{29}$ and then later further reduced to 11 teeth. With the 11-tooth main gear, maximum throttle yielded a safe 1800 RPM main rotor speed, and also supplied surprisingly good stability in the helicopter due to the high responsiveness of both the cyclic and collective controls. The increase in tail rotor RPM also yielded a very stable yaw control and aided the gyro in maintaining a heading without any drift, this had previously been an issue at lower RPM.

When the camera, the additional 11.1 volt, $800 \mathrm{mAh}$ LiPo battery and $2.4 \mathrm{GHz}$ transmitter were added to the helicopter it was again flight tested. The headspeed and stability proved satisfactory for image capture. The use of Velcro as an attachment medium for the camera, transmitters, and all other hardware proved to have an unexpected benefit: it acts as an excellent vibration dampener. It seems that one of the more interesting properties of hook and loop fasteners are that they allow a measurable amount of play while still holding with the same tenacity. It is only when the hook and loop connections are sheared apart that the holding power diminishes. Due to the generally low weight of the modeling equipment, and the high holding power of industrial Velcro, those limits are never experienced during routine flight. Moreover, the flight envelope of this particular

\footnotetext{
29 This too, produced a head-speed that was in excess of the maximum rating, but the RPM were lowered by running a lower throttle setting. This, however, is not an optimal situation for the speed controller and can cause other complications.
} 
camera-carrying helicopter is rather subdued, and therefore never approaches the separation strength required to peel apart the Velcro. Velcro, therefore, provides sufficient strength, while also allowing rapid prototyping of different layouts to help determine what will work best in any given scenario. 


\section{Structures for Consideration}

\subsection{Bridges}

Of most structure types, the one that can benefit most from remote aerial survey are bridges. A bridge, by its very nature, is designed to span terrain that would not otherwise make a suitable passageway for cars, trains or pedestrian traffic. Often, it is the only method of passage for kilometers and therefore a very vital piece of municipal infrastructure.

Due to span lengths and the relative height of many bridges and trestles, it is difficult to examine such structures without actually being physically on them. Figure 5-1 shows an extreme example of a trestle structure that would be difficult to examine in any detail without, perhaps walking or climbing onto the structure. On some bridges, it is not possible to examine the underside without repelling down the side, or climbing up from the bottom. In many cases the traffic-way of the span is lined with barbed wire, high railings, or other deterrents to prevent the public from climbing over the side. These safeguards hinder ready access to the structure and necessitate more work in removal of these obstacles for structural examination. 


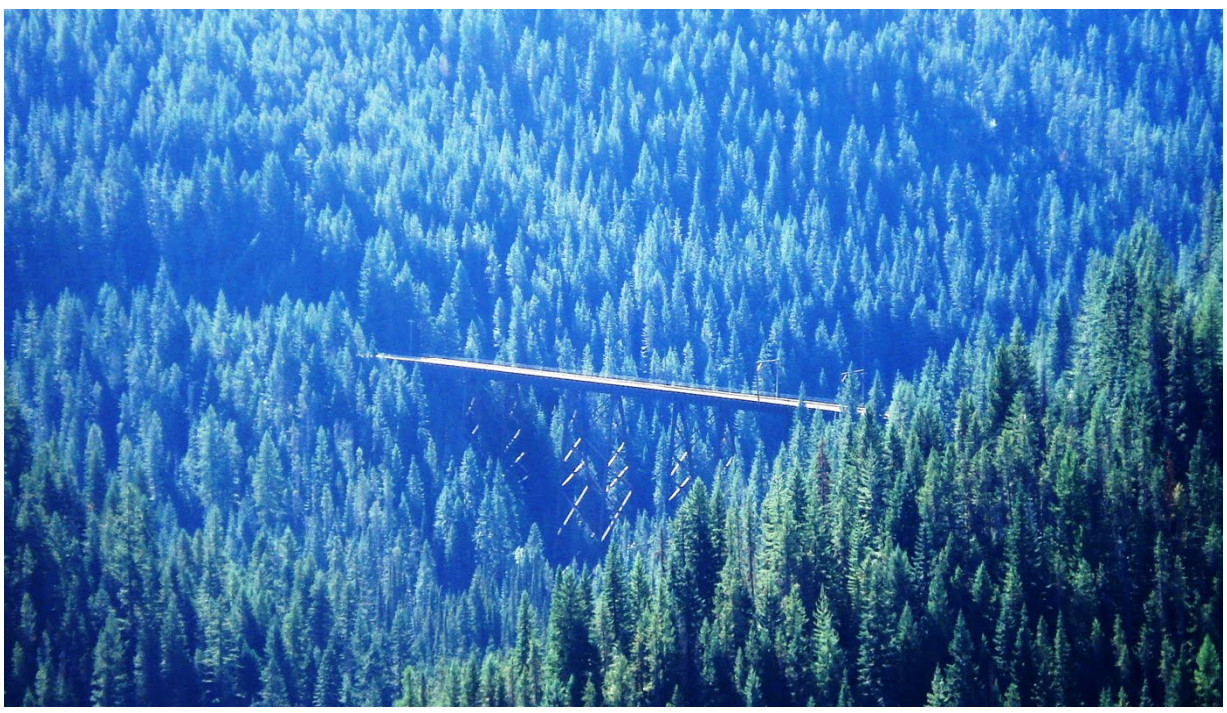

Figure 5-1 Kelly Creek Trestle, Montana, 260 meters long, 70 meters high. An example of a bridge structure that could benefit from quick, inexpensive, survey techniques.

\subsection{Power Transmission Towers}

While power lines are most often examined by full-scale helicopters with infrared cameras for detecting heat on the lines themselves, the height and relative remoteness of transmission towers make them prime candidates for survey from remotely controlled vehicles. Generally, transmission tower heights are between 15 and 55 meters $^{30}$, heights that are well within the flight envelope of model helicopters. Figure 5-2 shows a transmission structure of a kind that could be examined by a remotely controlled vehicle of the type described in this thesis, but would not be damaged should an accident or collision occur ${ }^{31}$.

\footnotetext{
30 There are some transmission towers that are considerably higher than 55 meters, but those are mostly located in Canada and Europe. With a larger class of model helicopter for visual piloting purposes, these could be surveyed also using the same method described herein.

31 The consequences of the tower being struck by a full scale helicopter are devastating, and would probably lead to loss of life and a disruption of service.
} 
While generally not a direct threat to humans, as a failing bridge or other structure might be, the impact of the failure of a transmission tower and the resultant loss of electrical transmission can have a far reaching effect and potentially life threatening consequences. Similar to bridges, the benefits of timely structural assessment are not to be undervalued.

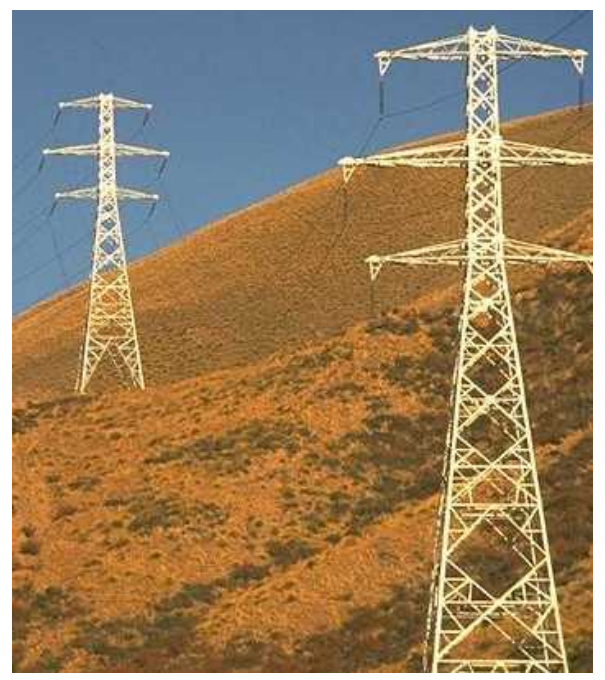

Figure 5-2 Three phase alternating current transmission towers.

\subsection{Smokestacks}

Although generally associated with a facility that is directly attached to, or in very close proximity to a manufacturing center or power generation facility, smokestacks are structures that can be easily compromised by earthquakes, and due to their closeness to people, deadly when they fail. Whereas these structures were at one time made of brick, they are now commonly constructed of pre-formed concrete, or sliding formwork construction. 
Due to their height, smokestacks can be challenging to examine, and while it would be daunting, if not entirely impossible, to examine the inside of the stack with a model helicopter, the close examination and photographing of key or suspicious points along the exterior of the structure can be accomplished from a safe, remote distance. Figure 5-3 illustrates a local example, smokestacks at the power plant in Morrow Bay that could be a beneficiary of such remote reconnaissance.

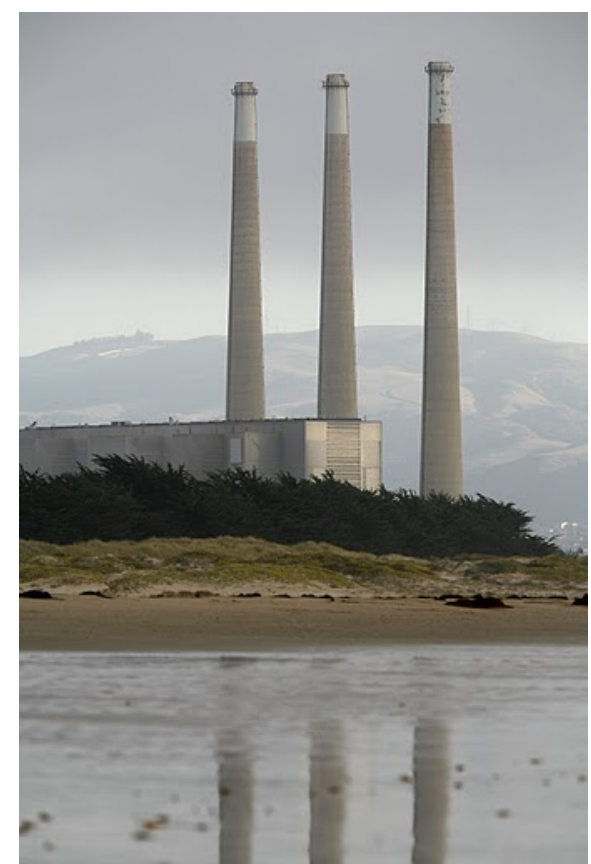

Figure 5-3 Morro Bay power plant with three 137 meter smokestacks.

\subsection{Other Structures}

Bridges, transmission towers, and smokestacks are just a few examples of suitable survey sites. Many types of structures could benefit from external observation. Thanks to their small size, environmentally clean operation, and low noise, electric remotely controlled helicopters can be employed even in densely 
populated areas, making them suitable for inner city observation of cell towers, radio masts, elevated walkways, external building facades, upper story windows, and rooftops, as examples. 


\section{Criteria for Test Survey Structure}

\subsection{Selection of Test Site}

Model helicopters, or any model vehicle used for survey, will only have utility if used in environments where observation by simpler methods is not possible. If a structure is three meters tall, for example, it is far simpler to walk up and observe the structure directly. Typically, the benefits of using a remotely controlled craft come from employing it as a set of eyes at a distance, and being able to move the user's viewpoint to examine the structure from angles that would otherwise not be accessible in a safe manner.

There are several structures in the general San Luis Obispo (California, Central Coast) area that are suitable test cases. After much consideration, the trestle located over Stenner Creek on the Cal Poly campus was chosen. Its age, structure type, height, and low traffic location constituted an ideal test site.

\subsection{About the Site}

The Stenner Creek Trestle was originally forged and partially assembled in Pittsburgh, Pennsylvania. It was deconstructed and shipped around the Cape Horn, then carried inland and assembled on location, in 1894. It is 235 meters long, and 25.9 meters high ${ }^{32}$. The aerial image, Figure $6-1$, shows the offset length of the trestle and its relationship to Stenner Creek and Stenner Creek road.

\footnotetext{
32 As noted from the Cal Poly Land website:

http://polyland.calpoly.edu/topics/technology/studentsites/2006b/Past/History\%20of\%20the\%20Trestle.htm
} 
Its location on Stenner Creek creates difficulty for observation from the ground, since it is inaccessible short of scaling the sides of the structure. There are trees and telegraph lines that inhibit direct views and make the structure difficult to approach with equipment. Wire railings line the sides. These rails are intended to keep people safely on the trestle. The track bed cantilevers out over the body of the structure making it difficult to study the underside of the structure without repelling down the side, climbing up from the bottom, erecting scaffolding, or bringing in heavy equipment that can carry human surveyors to the desired height and location.

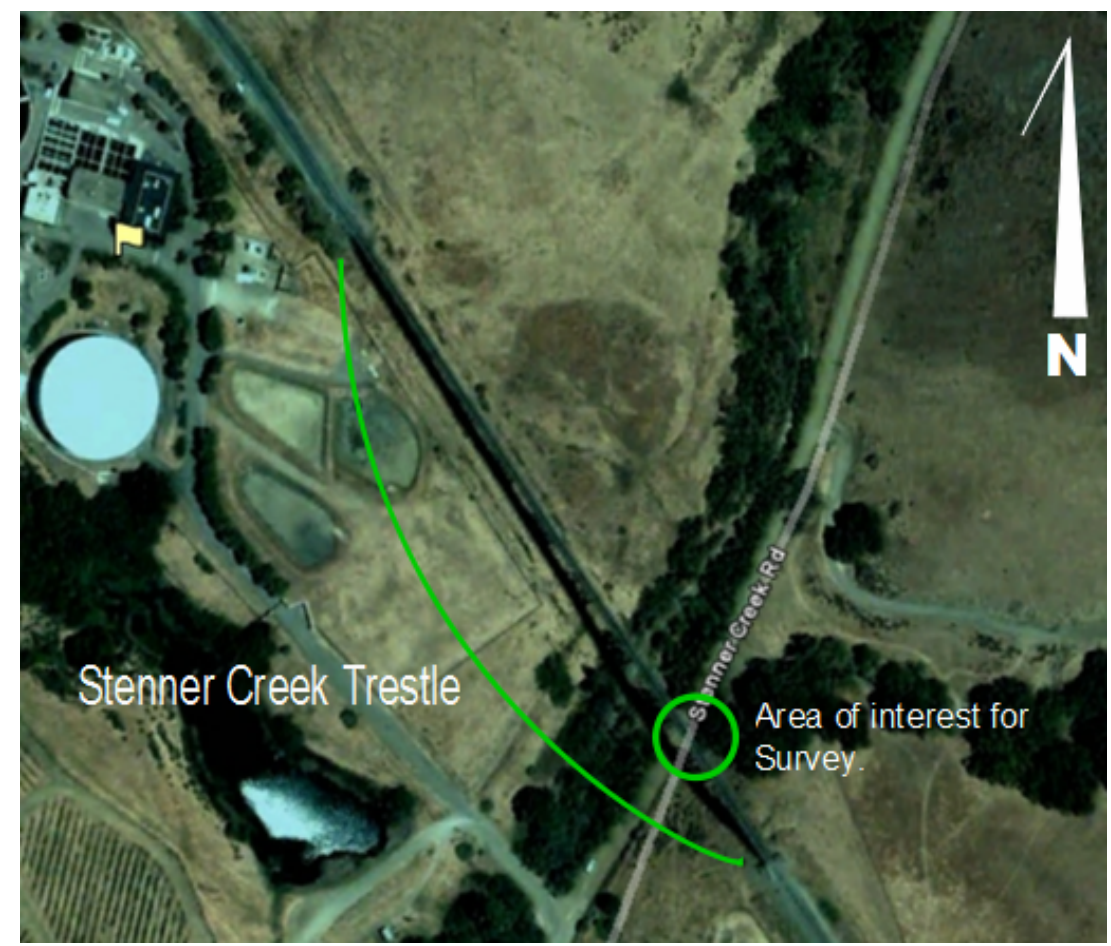

Figure 6-1 The Stenner Creek trestle with an indication of the area that was chosen for survey. Aerial picture (c) 2010 Google Earth. 
Due to the length of the structure and rough terrain, a smaller sub-section was chosen for testing, as seen in the circled area in Figure 6-1. Although the survey area is close to the road that runs alongside the creek under the trestle, the trestle itself is blocked from perpendicular viewing by trees and other obstacles.

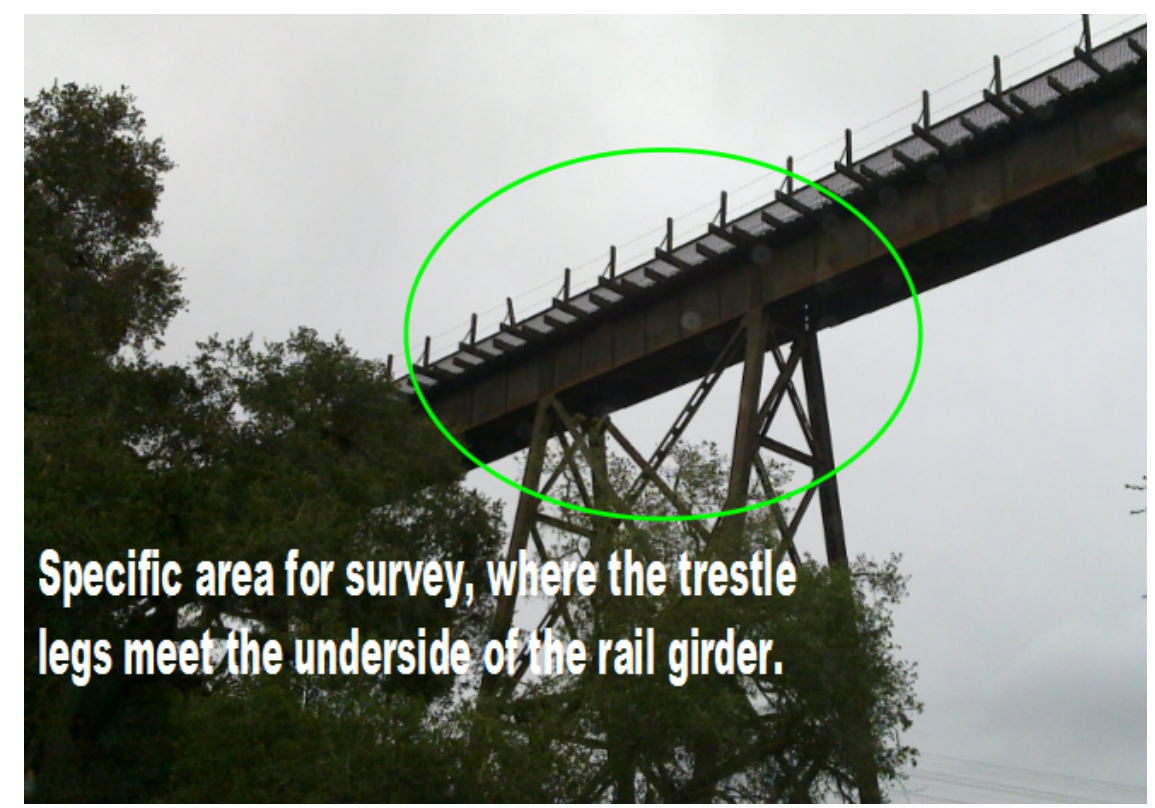

Figure 6-2 The connection point of the trestle and the girder is the focal point of study with the remote vehicle, as viewed to the south southwest, from road level.

The structure itself is made of riveted iron and steel. Noted in Figure 6-2 are four points where the supporting legs meet the double girders on which the rails rest. These points are of most interest from a safety standpoint. Failure of any one of these connections could lead to a derailment or other railway disaster. 


\section{Practical Helicopter Results}

\subsection{Initial testing}

The helicopter and camera platform proved to be quite stable. After taking time to ensure well-balanced main rotor blades, and a correct center of gravity (CG) when loaded with the various pieces of hardware, the platform was almost vibration free and reliable during consecutive test flights. Also, the remotely triggered camera operated as expected.

Consistently, the 10-minute test flights seemed to be more than adequately long, but when confronted with framing an actual test structure in true operating conditions the time passed much more quickly than anticipated. Due to the $50 \%$ safety factor on flight time, it was possible to maintain flight until the alarm had sounded, indicating the safe margin of flight time had elapsed and landing was required. Had the helicopter been a gas or nitromethane variant it might have been possible to have longer flights, but then other elements would have surfaced which, in retrospect, would have been potentially problematic, including: smoke and oil on the lens; vibration of the entire system; noise; and, probably many others that have yet to be considered.

The resolution of the camera is acceptable, but as noted in Section 4.4, technology continues to progress. One of the fastest areas of technological improvement has been digital imaging. With an upgraded camera capable of 
higher International Standards Organization (ISO) equivalency ${ }^{33}$ and with a higher pixel count the pictures would show more detail and be less blurred. A better imager will yield better results, and perhaps also alleviate the concerns regarding flying too close to the intended subject matter.

\subsection{Trestle results}

Due to the season, the first two scheduled flight tests at the Stenner Creek Trestle site were postponed. When, however, the weather cleared it was found that the proposed application did work as hypothesized. The helicopter flew over the trees surrounding the trestle, and was able to capture, via commands from the ground controller, images of the trestle. Figure 7-1 shows one of these pictures without any modification or image manipulation. Figure 7-2 illustrates that the image, with just some brightness adjustments can yield far more information to the viewer.

\footnotetext{
${ }^{33}$ ISO is a designation of how sensitive a film is. In the digital camera domain where there is no film it is referred to as "ISO equivalent". Unlike film, which must be physically removed and inserted, digital cameras allow the equivalency to be set via a menu system. New model cameras can emulate much higher speed film, allowing higher shutter speeds, or lower ambient light.
} 


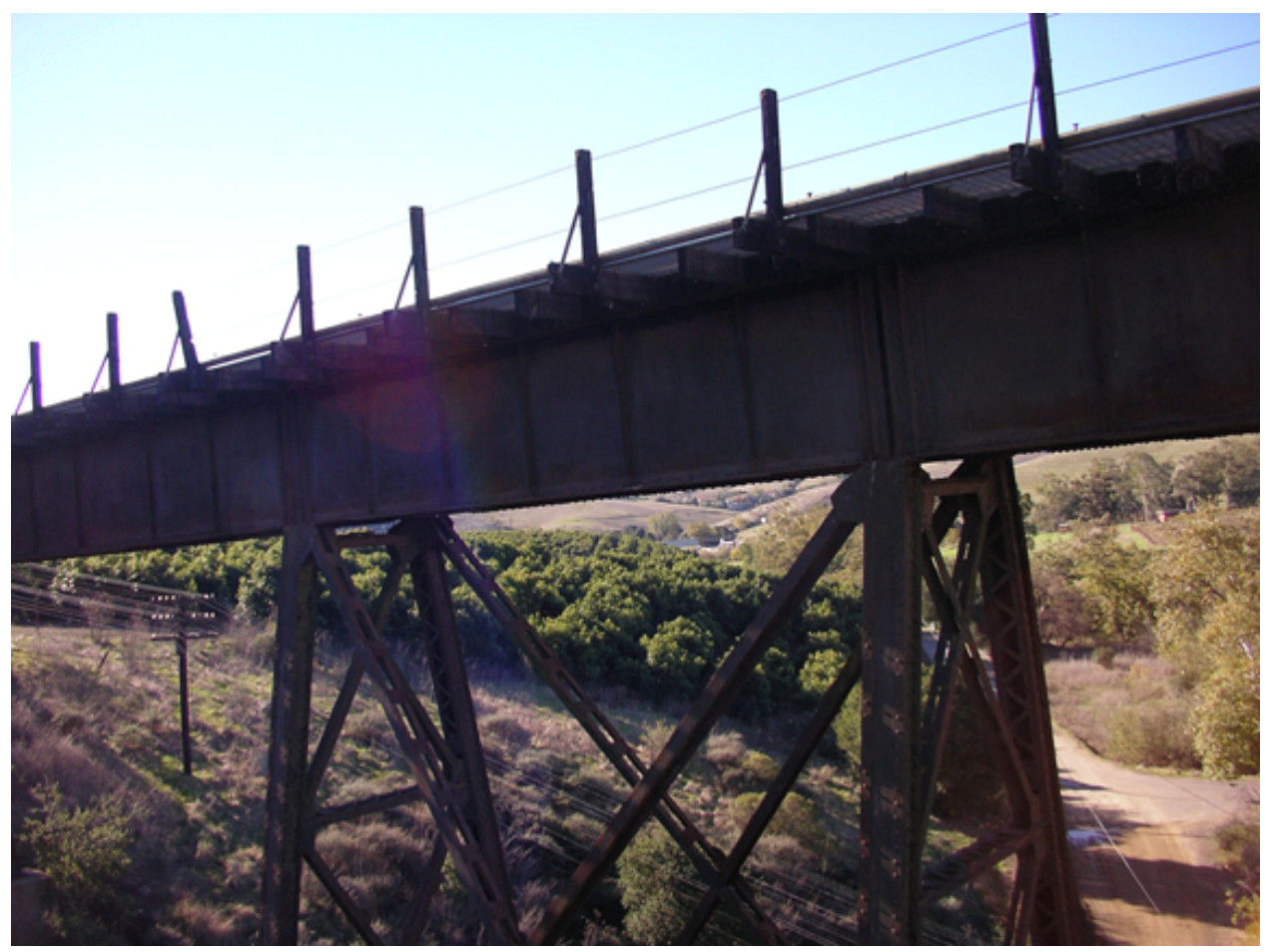

Figure 7-1 A picture taken from the photo platform prior to editing.

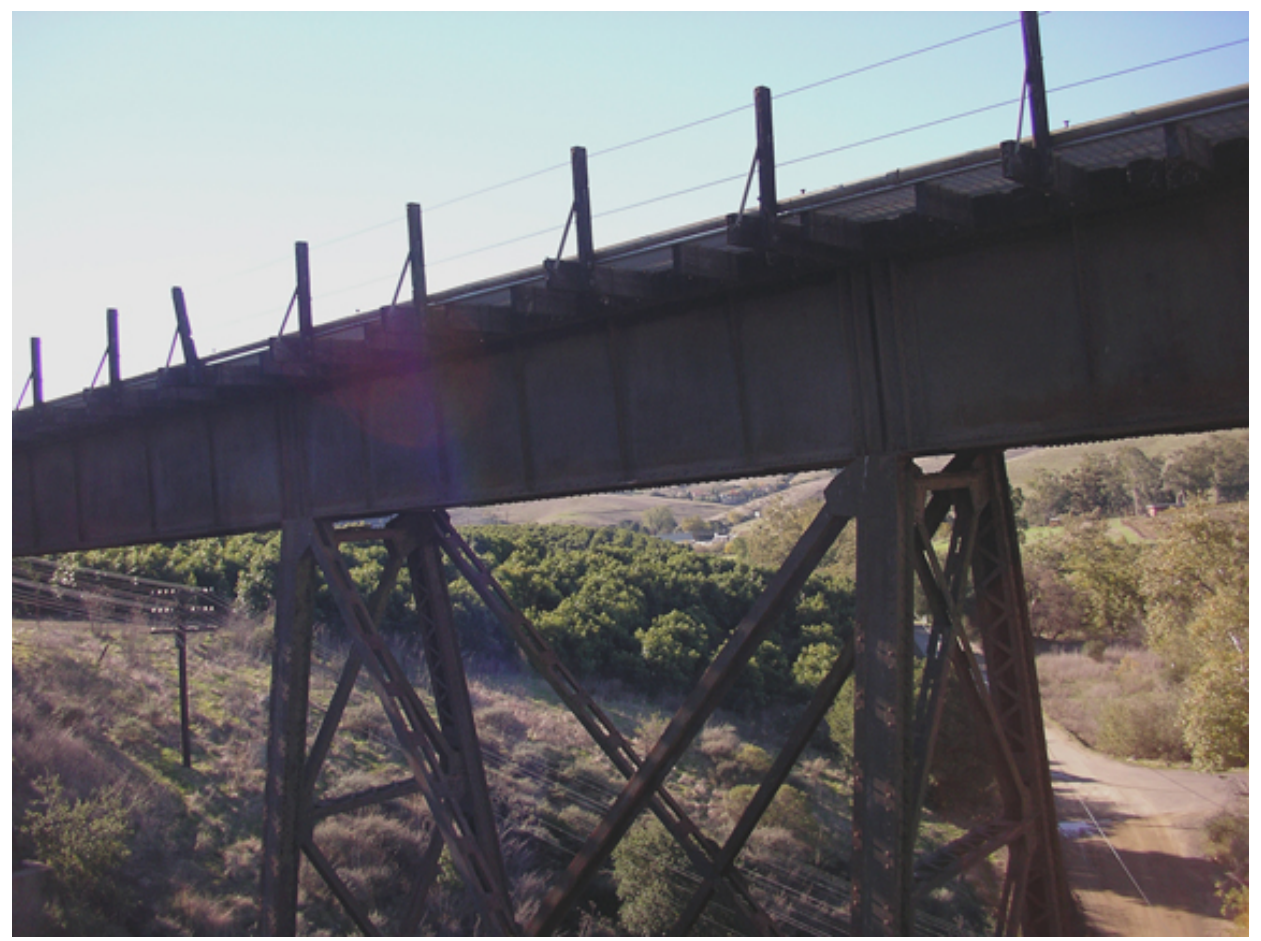

Figure 7-2 With Adobe Photoshop it is possible to enhance the picture so more detail is available. 


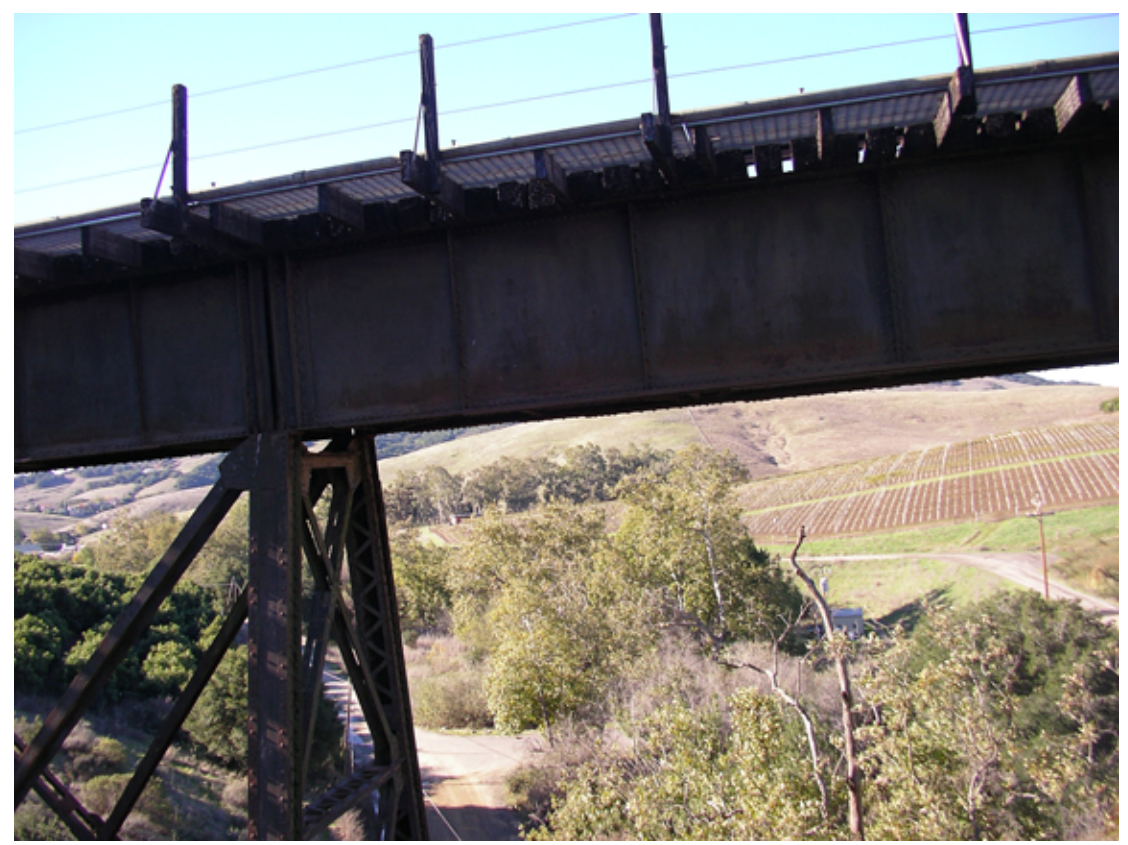

Figure 7-3 Another image with more exposed detail.

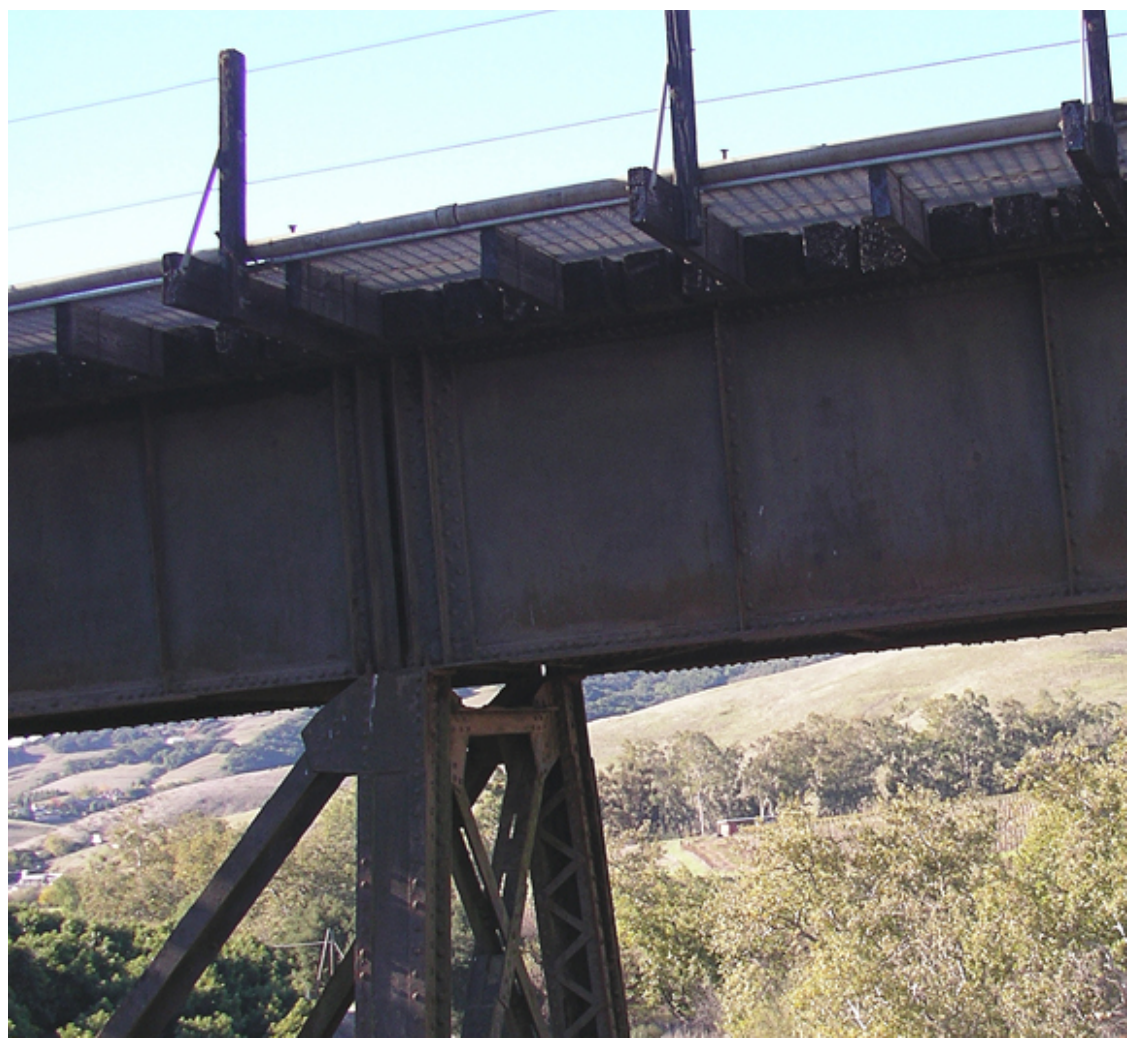

Figure 7-4 The view of a cropped area of Figure 2-3 with some slight Photoshop contrast and sharpening adjustments. 
Figure 7-3 shows another image capture with more detail available. It is still difficult to see the details of the rivets, but a cropped and brightened version, Figure 7-4, shows rivets, and it is possible to see cracks in the ends of the wood decking above the ironwork. Areas of rust and corrosion are visible, as are bird droppings. Finally, Figure $7-5$ is yet one more view of the girder section where, in Figure 7-6 after cropping and brightening, it is possible to view the fiber optic cable suspended from the underside of the wooden decking and above the girder, as well as more rust and weather damage to the wood decking.

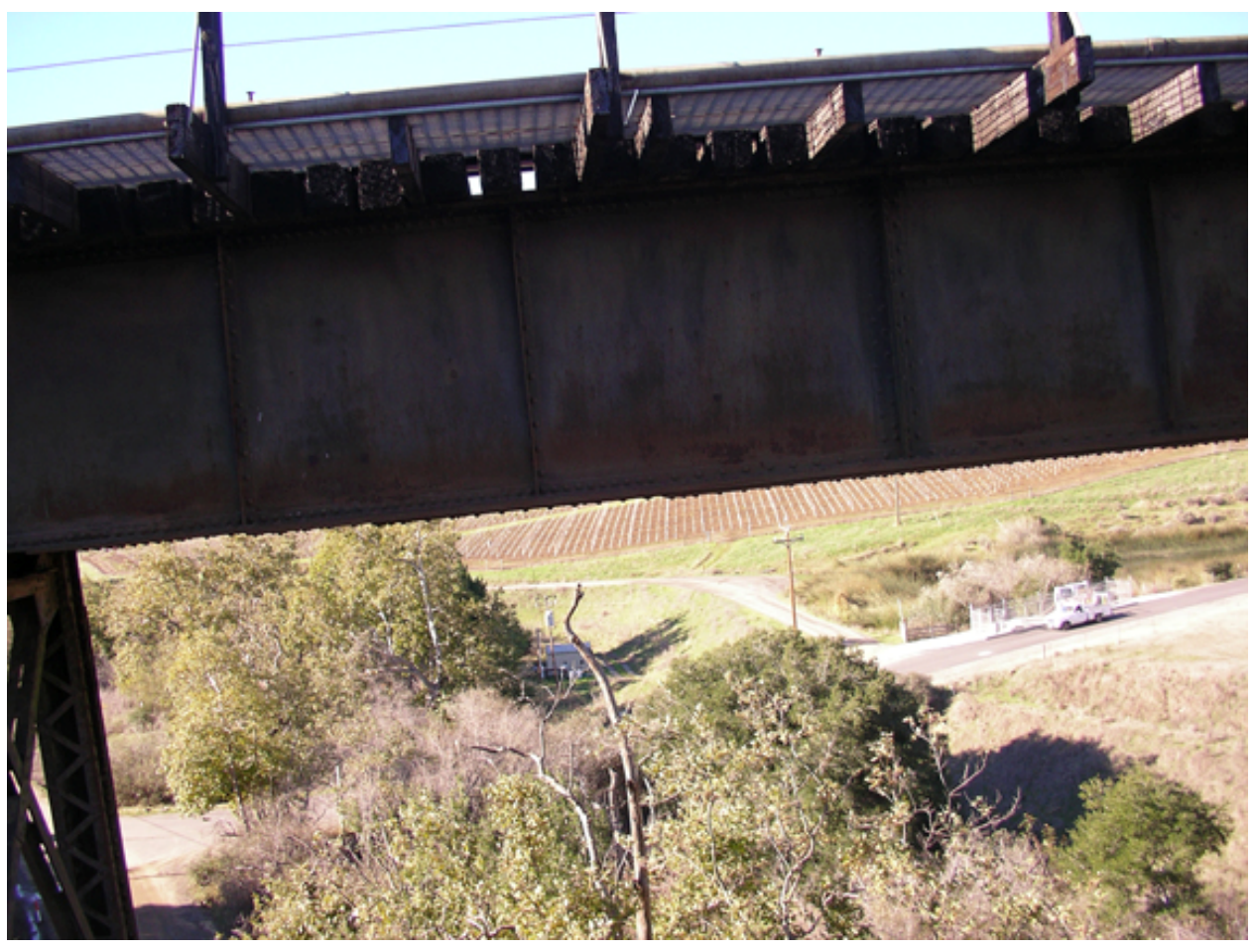

Figure 7-5 Another example image with better exposure. 


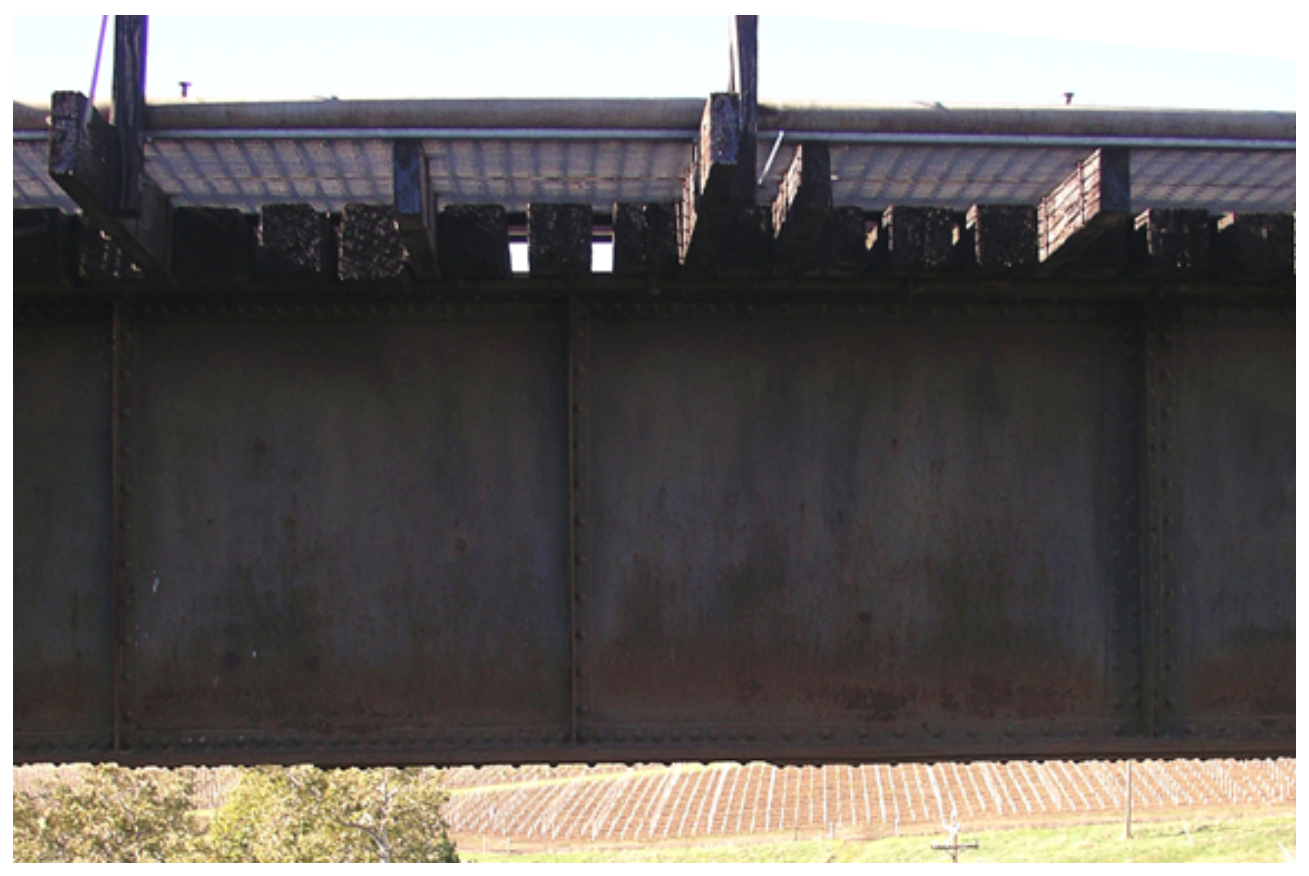

Figure 7-6 A close up cropped and rotated version of Figure 7-5, note the orange fiber optic cable conduit on the upper left center. 


\section{Conclusion}

\subsection{Changes In Technology Over Time}

During the six-year duration of this thesis, there have been changes in technology and regulations that have altered the landscape of this area of study. Chiefly, these are advancements and developments in technology that aid model airplane flying. Seven years ago a model helicopter "flight augmentation system" was not quite a reality, or at least not available on the open market. Today, a similar system costs about (US - 2010) \$140 and can be purchased on the Internet. The size of servos has decreased; the torque produced by those smaller servos has increased to beyond the specifications of the larger outdated servos they replaced. Battery technology has progressed from leading edge technology, to something that is now ubiquitous and available in every hobby shop.

During this same period the Federal Aviation Administration (FAA) has taken a more critical view of model planes with cameras mounted on them. Initially it was an unlimited area of development and experimentation. However, since the risk of terrorism became an ever-present fear, the federal government has begun to slowly tighten the control on model flight ${ }^{34}$. At one point about half way through the design phases of this project it became unclear if the vehicle could be legally

\footnotetext{
34 Although terrorism has always been an issue, the terrorist attack of 11 September, 2001 brought the microscope of inspection into the arena of technology, and this includes model "toys", whose sophistication and precision are exceedingly high, and where generational cycles are measured in months, not years.
} 
tested. Thankfully, the rules have not yet become more stringent ${ }^{35}$, and therefore testing then and now has been allowed to continue. The final rules have yet to be established and therefore planes with cameras mounted can still be flown under guidelines of general model aviation. ${ }^{36}$

The researcher's initial testing of camera/transmitter combinations was from a side-mounted camera on a model plane ${ }^{37}$. The results were well worth the effort; the assembly performed well in several different settings and locations. Since a stable platform that could also hold a static position was required, it became clear that a helicopter is most likely the most appropriate platform. The quadcopter was, six years ago, still a cutting edge, undefined entity, whose computing requirements for flight augmentation had yet to be developed. It has clearly emerged as a viable platform, but is still in the development phases.

\subsection{Issues}

The chief issue and major hurdle to be overcome in order to achieve better results is imaging quality. There is no doubt that a helicopter mated with a flight augmentation system is quite capable of carrying the required camera and telemetry payload. It is also quite evident that it can fly within the wind ranges specified, and it can also take pictures. With a better camera system it is

\footnotetext{
35 Thanks, in no small part, to the slow speed of bureaucracy. It is this researcher's opinion that changes will come, favoring the larger corporations, not the garage designers and innovators.

${ }^{36}$ Some caveats still apply when trying to sell images, or when flying from a "first person view" (FPV) perspective using only the camera for visual guidance. While in FPV flight mode it is required to have a spotter, in the event the vehicle goes out of control or the signal is lost. The spotter should be capable of taking control of the craft in the event the primary pilot loses control.

37 The Multiplex EasyStar was the platform used for testing as it is inexpensive, durable and simple to work with.
} 
possible to fly farther from the target in inclement weather conditions and still obtain a satisfactory result without jeopardizing safety.

Controlling and aiming the helicopter/camera combination became much more challenging when flying from a distance. This, in part, is due to the inability to see and react to small movements in the hovering helicopter that are due to environmental stimuli ${ }^{38}$. As the vehicle is farther away, the visual angular change is much less, and therefore it is not possible to react and counter the positional change until the helicopter has covered a greater distance. While the flight augmentation system is taking care of the physics of the helicopter, it is not holding it in the appropriate relative position. Systems do exist that can maintain position via a global positioning system (GPS) signal, but those are much more costly and do not fit the price range established at the outset of the research.

\footnotetext{
${ }^{38}$ In this case those stimuli are wind and/or turbulence caused by wind blowing around or over objects.
} 


\section{Future Work}

\subsection{Organization of Vehicle Operators}

Despite some of the shortcomings exhibited in a survey vehicle, the researcher feels that it would not be detrimental for a municipality to have machines, such as the one described in this thesis, available for use in the event of a natural disaster. The vehicles would require operators. Initially it was unclear how that could be organized, but then the operational structure under which the amateur radio operators function proved to be a fairly mature and useful framework to develop in this context.

Through working contracts with model pilots it might be possible for a municipality to create working agreements with model clubs such that they have one or two of these machines (or an analogous system) available to the community in exchange for, perhaps in city or county flying areas. This would be a favorable situation, as model clubs nation-wide have an increasingly difficult time finding and/or keeping flying sites, and municipalities should not be in the business of keeping and maintaining model aircraft.

\subsection{Available User Base in Model Aircraft Flying}

The Academy of Model Aeronautics (AMA) maintains a list of membership from their 10 districts in the United States (U.S.). The AMA is the definitive model flying organization of the United States with a membership of roughly 150,000 . 
AMA pilots are located across the country in 12 districts with a total number of 2344 clubs. See the Table 9-1 for the distribution of clubs across the U.S.

Table 9-1 Number of R/C Flying Clubs Distributed across the U.S.

\begin{tabular}{|l|c|}
\hline \multicolumn{1}{|c|}{ AMA District } & Number of Clubs \\
\hline 1; CT, MA, ME, NH, RI, VT & 117 \\
\hline 2; NJ, NY, PA & 185 \\
\hline 3; FL, IN, KY, MI, OH, PA, WV & 263 \\
\hline 4; DE, MD, NC, SC, VA & 177 \\
\hline 5; AL, FL, GA, MS, PR, SC, TN & 313 \\
\hline 6; AR, IL, KY, MO, OH & 259 \\
\hline 7; IA, IL, MI, MN, NE, TX, WI & 250 \\
\hline 8; AR, KS, LA, NM, OK, TX & 234 \\
\hline 9; CO, KS, MO, ND, NE, SD, WY & 122 \\
\hline 10; AZ, CA, GU, HI, NV, UT & 283 \\
\hline 11; AK, CA, ID, MD, MT, OR, WA & 140 \\
\hline \multicolumn{1}{|c|}{ Total Flying Clubs } & $\mathbf{2 , 3 4 3}$ \\
\hline
\end{tabular}

\subsection{Total Automation}

The final step in this process would be to totally automate the helicopter, again using off-the-shelf components. It would be far superior to have the helicopter fly itself to the desired location while holding the desired heading and altitude. Currently these technologies are still immature, but do exist in the hobbyist world. The author believes that in a few years these technologies are likely to be available in the form of off-the-shelf systems that are priced in a range that meets the requirements laid out in this thesis. 
Vehicle automation would also resolve the issues associated with control difficulties that present themselves when flying a model at a distance. Since the vehicle will have full knowledge of its location, and the conditions that it is flying in, there should be inherently more stability and better resolution in positioning. That kind of control, combined with four or five times the resolution present in this early demonstration model should have the capability to take very fine images from almost any position. 


\section{List of References}

Croucher, P (2007); "Private Helicopter Pilot Studies, JAA Version", Lulu.com Publishing (pp. 2-20)

Federal Aviation Administration (FAA) (2007); "Rotorcraft Flying Handbook", Skyhorse Publishing, Inc. New York, U.S. (pp. 8-2)

Johnson, W (1994); "Helicopter Theory", Princeton University Press, New Jersey, U.S. (pp. 5-10, 149-155)

Leishman, J (2006); "Princliples of Helicopter Aerodynamics"; Cambridge University Press, Cambridge, U.K. (pp. 271)

Messiter, M (2007); "Helicopters A Guide for Beginners"; Traplet Publications Ltd, Worcestershire, U.K.. (pp. 41)

Padfield, G (2007); "Helicopter Flight Dynamics: The Theory and Application of Fliying Qualities and Simulation Modelling", Blackwell Publishing, Oxford, U.K. (pp. 16-21)

Zischinsky,T, Dorfner,L, and Rottensteiner, F (2000); "Application of a New Model Helicopter System in Architectural Photogrammetry", IAPRS, VOL 33, Amsterdam, The Netherlands. 